\title{
Extended multi-scalar field theories in $(1+1)$ dimensions
}

\author{
A. R. Aguirre ${ }^{\mathrm{a}}$, , E. S. Souza ${ }^{\mathrm{b}}$ \\ Instituto de Física e Química, Universidade Federal de Itajubá, Av. BPS 1303, Itajubá, MG CEP 37500-903, Brazil
}

Received: 23 October 2020 / Accepted: 30 November 2020 / Published online: 11 December 2020

(C) The Author(s) 2020

\begin{abstract}
We present the explicit construction of some multi-scalar field theories in $(1+1)$ dimensions supporting BPS (Bogomol'nyi-Prasad-Sommerfield) kink solutions. The construction is based on the ideas of the so-called extension method. In particular, several new interesting two-scalar and three-scalar field theories are explicitly constructed from non-trivial couplings between well-known one-scalar field theories. The BPS solutions of the original one-field systems will be also BPS solutions of the multi-scalar system by construction, and therefore we will analyse their linear stability properties for the constructed models.
\end{abstract}

\section{Introduction}

Recently, there has been a great deal of interest in investigating multi-scalar field theories in $(1+1)$ dimensions, which support kink solutions obeying first-order BPS equations [1-7]. Interesting applications have been found mainly in the context of cosmological models [8-12], in the study of some aspects of self-duality in generalised BPS theories [13-15], and also in connections with several others relevant subjects [16-28]. Among others topological defects [29,30], the study of kink solutions in scalar field theories are of great importance in several areas of the modern theoretical physics $[31,32]$. They are non-trivial static solutions of the non-linear field equations with finite energy satisfying especially boundary conditions, which usually describe models that exhibit spontaneous symmetry breaking. However, finding out analytically such kind of solutions for a given field theory is in general a quite difficult task, especially for the case of multifields systems. In some cases thought, it is possible to make use of some indirect methods to find analytical solutions, e.g. the so-called trial orbit method [33-36], which allow us decouple the field equations by introducing very specific

\footnotetext{
a e-mail: alexis.roaaguirre@unifei.edu.br (corresponding author)

b e-mail: edson_souza@unifei.edu.br
}

orbit equations of the form $\mathcal{O}\left(\phi_{1}, \ldots, \phi_{n}\right)=0$, that is constraints in the target space. Although useful, this method has been shown not so efficient when one is looking for new analytical multi-scalar models.

In that scenario, a simplifier tool in searching for kink solutions is provided by the so-called BPS (Bogomol'nyiPrasad-Sommerfield) method [37,38], which allows to find solutions from first-order differential (BPS) equations instead of second-order Euler-Lagrange equations. BPS solutions correspond to static configurations of minimal energy. Although simpler, the problem of solving analytically firstorder differential coupled equations is still not easy, and then it is necessary to use additional procedures to sort it out.

In this work, we will use the extension method, originally proposed in $[1,2]$, to systematically construct several new multi-scalar field theories in $(1+1)$ dimensions supporting BPS states, starting from a system of several one-scalar models. The basic ingredients in the construction are the socalled deformation functions and its inverses [39-42], which provide suitable links between the fields to be coupled. In addition, the method has the nice advantage that the BPS solutions of the one-field systems are also solutions for the multi-scalar system.

We aim that the new models constructed in the present work could improve the knowledge and understanding of the analytical solutions of multi-scalar systems, and believe that they have potential applications to cosmological models, to the study of kink scattering process of multi-solitons, and also to analyse integrability and self-duality properties in the multi-scalar-models. For that reason, special attention will be given to the theories with periodic potentials with infinitely degenerate vacua, as is the case of sine-Gordon model, or even more exotic models as the one studied in $[43,44]$.

This paper is organized as follows. In Sect. 2, we briefly review some basics aspects of scalar BPS theories, introducing the superpotential function, or sometimes called the prepotential function [13], as the key ingredient of the whole construction. In Sect. 3, we present the main ideas of the 
deformation procedure, and then we apply it to obtain several examples of deformed theories. In Sect. 4, we introduce the extension method and construct several new interesting two-scalar field theories. The linear stability of the BPS solutions for these new models will be discussed in Sect. 5. In Sect. 6, we construct some new three-scalar fields extended models by applying a straightforward generalization of the extension method for three-field systems [2], and also analyse the linear stability of their BPS solutions. Final remarks and comments of our work are presented in Sect. 7. Finally, in Appendix A, we have summarized some basics features of the underlying exactly solvable potentials which will be appear along the linear stability analysis.

\section{General settings}

Let us start considering theories with $n$ real scalar fields $\phi_{a}(x, t), a=1, \ldots n$, in $(1+1)$ dimensions described by the following Lagrangian, ${ }^{1}$

$\mathcal{L}=\frac{1}{2} \sum_{a=1}^{n}\left(\partial_{\mu} \phi_{a}\right)^{2}-V(\phi)$.

The corresponding field equations for $\phi_{a}(x, t)$ are given by

$\partial_{t}^{2} \phi_{a}-\partial_{x}^{2} \phi_{a}+\frac{\partial V}{\partial \phi_{a}}=0$,

and for the static configurations $\left(\partial_{t} \phi_{a}=0\right)$, we get

$\phi_{a}^{\prime \prime}(x)=\frac{\partial V}{\partial \phi_{a}}$,

where we are using standard conventions $\phi_{a}^{\prime} \equiv \frac{d}{d x} \phi_{a}$. These equations can be rewritten as follows,

$\frac{1}{2}\left(\phi_{a}^{\prime}\right)^{2}=\int \frac{\partial V}{\partial \phi_{a}} d \phi_{a}$.

It is worth pointing out that there is no summation assumed in Eq. (4). The corresponding energy functional for the static configurations reads,

$E[\phi]=\int_{-\infty}^{\infty} d x\left(\frac{1}{2} \sum_{a=1}^{n}\left(\phi_{a}^{\prime}\right)^{2}+V(\phi)\right)=2 \int_{-\infty}^{\infty} d x V(\phi)$.

Finite energy configurations require existence of the boundary conditions $\phi_{a}^{\prime}(\infty) \rightarrow 0$, and a potential possessing at least one vacuum value, $V(\bar{\phi})=0$, such that $\phi_{a}( \pm \infty) \rightarrow$ $\bar{\phi}_{a}^{ \pm}$. When two or more minima exist, then the potential supports topological configurations connecting two adjacent minima $\bar{\phi}^{-}$and $\bar{\phi}^{+}$. Now, by introducing a smooth function

\footnotetext{
${ }^{1}$ Here $\mu=\{0,1\}$, with metric convention $\eta_{\mu \nu}=\operatorname{diag}(+1,-1), \partial_{\mu} \equiv$ $\frac{\partial}{\partial x^{\mu}}, x^{0}=t, x^{1}=x$, in natural units.
}

of the scalar fields $W^{(n)}(\phi)$, sometimes named superpotential or pre-potential [13], the potential $V$ can be written as,

$V(\phi)=\frac{1}{2} \sum_{a=1}^{n}\left(W_{\phi_{a}}^{(n)}\right)^{2}$,

where $W_{\phi_{a}}^{(n)}$ stands for $\frac{\partial W^{(n)}}{\partial \phi_{a}}$. Then, the field equations can be rewritten as a set of coupled first-order differential equations,

$\phi_{a}^{\prime}= \pm W_{\phi_{a}}^{(n)}$,

with energy given by,

$E=\left|W^{(n)}\left(\bar{\phi}^{+}\right)-W^{(n)}\left(\bar{\phi}^{-}\right)\right|=E_{\mathrm{BPS}}$.

The solutions of the first-order differential equations (7) with non-zero energy (8) are named BPS states. These minimum energy static configurations are also solutions of the second-order differential equations (3), which can be understood from the self-duality properties of the BPS theories as claimed in [13]. In fact, for a given field theory, the Bogomol'nyi bound (8) only depends on the boundary conditions, and not on the field configuration, which means that $E_{\mathrm{BPS}}$ is a homotopy invariant, that is invariant under any smooth deformation of the field configurations. These interesting properties makes BPS states so attractive, and it will be the main goal of our work to look for them.

\section{Deforming one-scalar field theories}

Recently, it has been proposed an interesting procedure to generate infinite families of one-field theories with topological (kink-like) or non-topological (lump-like) solutions, which is now referred as deformation procedure $[39,40]$. The main idea is to start from a given "seed" one-scalar field theory possessing static solutions, and then perform a field transformation on the target space to obtain a new one-scalar field theory that also supports static solutions. In particular, we will focus in theories supporting BPS solutions.

Let us start from a one-scalar field model described by the Lagrangian,

$\mathcal{L}=\frac{1}{2} \partial_{\mu} \phi \partial^{\mu} \phi-V(\phi), \quad$ with $V(\phi)=\frac{1}{2}\left(W_{\phi}^{(1)}\right)^{2}$,

which supports BPS solutions satisfying the first-order differential equation,

$\phi^{\prime}=W_{\phi}^{(1)}(\phi)$.

Now, we introduce an invertible smooth function $f$ on the target space, called the deformation function, such that

$\phi(x)=f(\varphi(x))$,

where $\varphi$ is a new (deformed) scalar field. This function also allows us to introduce a new (deformed) one-field model 
described by the following Lagrangian,

$\widetilde{\mathcal{L}}=\frac{1}{2} \partial_{\mu} \varphi \partial^{\mu} \varphi-\widetilde{V}(\varphi), \quad$ with $\widetilde{V}(\varphi)=\frac{1}{2}\left(\widetilde{W}_{\varphi}^{(1)}\right)^{2}$,

which satisfies the first-order equation,

$\varphi^{\prime}=\widetilde{W}_{\varphi}^{(1)}(\varphi)$,

providing that the two potentials are related to each other through the deformation function as follows,

$\widetilde{V}(\varphi)=\frac{1}{f_{\varphi}^{2}} V(\phi \rightarrow f(\varphi))$,

where $f_{\varphi}=\frac{d f}{d \varphi}$. This also implies that the two superpotentials are related in the following form,

$W_{\phi}^{(1)}(\phi \rightarrow f(\varphi))=W_{\phi}^{(1)}(\varphi)=f_{\varphi} \widetilde{W}_{\varphi}^{(1)}(\varphi)$.

It is worth noting that the static solutions for both scalar fields are related by Eq. (11), and then by replacing in the first-order differential equation, we find that they also satisfy the following important constraint,

$\frac{d \phi}{d \varphi}=\frac{W_{\phi}^{(1)}(\phi)}{\widetilde{W}_{\varphi}^{(1)}(\varphi)}$.

This relation between the fields will play a central role in constructing multi-scalar field theories supporting BPS kinklike solutions. In fact, this relation has been already used in [45] for studying systems of two coupled fields in $(1+1)$ dimensions through orbit equation deformations.

Let us now consider a few interesting examples to illustrate the deformation procedure. First of all, we start with the standard $\phi^{4}$ model [42], whose potential can be written as

$V(\phi)=\frac{\alpha^{2}}{2}\left(1-\phi^{2}\right)^{2}$,

where $\alpha>0$, is a real dimensionless parameter. This potential satisfies the first-order differential equation

$\phi^{\prime}=W_{\phi}^{(1)}(\phi)=\alpha\left(1-\phi^{2}\right)$,

and supports the following static solution,

$\phi(x)=\tanh (\alpha x)$.

Now, in order to obtain the deformed model, we consider the following function,

$\phi=f(\varphi)=|\varphi|-1$.

After using the deformation function, we obtain that the deformed potential

$\widetilde{V}(\varphi)=\frac{\alpha^{2}}{2} \varphi^{2}(2-|\varphi|)^{2}$, describes the so-called $\varphi^{6}$-like model [41]. The corresponding the first-order differential equation is given by,

$\varphi^{\prime}=\widetilde{W}_{\varphi}^{(1)}=\alpha \varphi(2-|\varphi|)$,

with the following topological solutions

$\varphi_{ \pm}(x)= \pm(1+\tanh (\alpha x))$,

which are quite similar to the solutions of the standard $\phi^{6}$ model [42]. This model possesses three minima at the values $\bar{\varphi}=\{0, \pm 1\}$, and supports two symmetric BPS sectors [41]. Interestingly, this example shows us that the deformation procedure can change the number of vacua of the seed model, and consequently changing the number of topological sectors.

As a second example, let us consider again the $\phi^{4}$ model as the seed model, with superpotential given by (18), and introduce the following periodic deformation

$\phi=f(\chi)=\sin (\beta \chi)$,

where $\chi$ is the new deformed field. The corresponding deformed model describes the sine-Gordon model given by the following first-order equation

$\chi^{\prime}=\widetilde{W}_{\chi}^{(1)}(\chi)=\frac{\alpha}{\beta} \cos (\beta \chi)$.

This very well-known model has infinite degenerate vacua at the values $\bar{\chi}_{k}=\left(k-\frac{1}{2}\right) \frac{\pi}{\beta}$, with $k \in \mathbb{Z}$, and correspondingly an infinite number of equivalent topological sectors. Connecting the minima $\bar{\chi}_{0}$ and $\bar{\chi}_{1}$, its static solution can be written as follows,

$\chi(x)=\frac{1}{\beta} \arcsin (\tanh (\alpha x))$.

As a last example, let us consider the bosonic exotic scalar model (E-model) investigated in $[43,44]$ as our seed model, which is described by the following first-order field equation,

$\eta^{\prime}=W_{\eta}^{(1)}=\alpha(1+\eta) \cos \left(\frac{1}{2} \ln (1+\eta)^{2}\right)$.

This model also has infinitely degenerate trivial vacua at the points $\bar{\eta}_{k}=-1+e^{\left(k-\frac{1}{2}\right) \pi}$, with $k \in \mathbb{Z}$. However, in this case the infinite number of BPS sector are not equivalent, since the BPS energy depends on the topological sector. A simple kink-like solution for this model connecting the vacua $\bar{\eta}_{0}$ and $\bar{\eta}_{1}$, can be written as follows,

$\eta(x)=\exp (\arctan (\sinh (\alpha x)))-1$.

Now, by considering the following deformation function,

$\eta=f(\chi)=\left(e^{\beta \chi}-1\right)$,

we get the sine-Gordon model, which has been already described in Eq. (25). 


\section{Constructing two-scalar fields models}

Let us now describe the method to construct two-scalar field theories from one-scalar field theories. To do that, we will use the deformation method introduced in the last section. The starting point is the first-order equation for the seed onescalar field model,

$\phi^{\prime}=W_{\phi}^{(1)}(\phi)$,

which supports static solutions. Now, by introducing a deformation function, i.e. $\phi=f(\varphi)$, we can rewrite Eq. (30) in two different (but equivalent) ways, namely

$\phi^{\prime}=W_{\phi}^{(1)}(\varphi)$, and $\phi^{\prime}=W_{\phi}^{(1)}(\phi, \varphi)$,

where we have made full use of the function $\phi \rightarrow f(\varphi)$ in the first expression in order to make $W_{\phi}^{(1)}$ a function depending only on $\varphi$, while in the second expression we have made partial use of this function in order to make $W_{\phi}^{(1)}$ a function depending on both fields $\phi$ and $\varphi$. Of course, there is an ambiguity in obtaining the last expression since it would depend on how this "lifting" from $\phi$-space to $(\phi, \varphi)$-space is made. Then, there will be an infinite number of resulting models once we chose the form of $W_{\phi}^{(1)}(\phi, \varphi)$. Some of these models would be trivial, and some of them even do not longer support kink-like solutions. However, our main goal here is to construct models that do support BPS solutions, and that will be reach by choosing carefully that form.

Taking into account that the deformed field $\varphi$ also satisfied a first-order differential equation,

$\varphi^{\prime}=\widetilde{W}_{\varphi}^{(1)}(\varphi)$,

the same procedure can be applied in order to rewrite the equation as follows,

$\varphi^{\prime}=\widetilde{W}_{\varphi}^{(1)}(\phi), \quad$ and $\varphi^{\prime}=\widetilde{W}_{\varphi}^{(1)}(\phi, \varphi)$,

where again there have been both a full as well as a partial use of the deformation (inverse) function. Note also that now the Eq. (16) can be rewritten in several different ways. In fact, in order to proceed with the extension method, we define the new two-fields superpotential through the following ansatz,

$$
\begin{aligned}
W_{\phi}^{(2)}(\phi, \varphi)= & a_{1} W_{\phi}^{(1)}(\varphi)+a_{2} W_{\phi}^{(1)}(\phi, \varphi)+a_{3} W_{\phi}^{(1)}(\phi) \\
& +p_{1} g(\varphi)+p_{2} g(\phi, \varphi)+p_{3} g(\phi), \\
W_{\varphi}^{(2)}(\phi, \varphi)= & b_{1} \widetilde{W}_{\varphi}^{(1)}(\varphi)+b_{2} \widetilde{W}_{\varphi}^{(1)}(\phi, \varphi)+b_{3} \widetilde{W}_{\varphi}^{(1)}(\phi) \\
& +q_{1} \tilde{g}(\varphi)+q_{2} \tilde{g}(\phi, \varphi)+q_{3} \tilde{g}(\phi),
\end{aligned}
$$

where for consistency, the parameters $a_{i}, b_{i}, p_{i}$, and $q_{i}$ with $i=1,2,3$, must satisfy the following constraints

$$
\sum_{i=1}^{3} a_{i}=\sum_{i=1}^{3} b_{i}=1, \quad \text { and } \quad \sum_{i=1}^{3} p_{i}=\sum_{i=1}^{3} q_{i}=0,
$$

and $g$ and $\tilde{g}$ are arbitrary functions required for the consistency conditions, which can be written as follows,

$W_{\phi \varphi}^{(2)}(\phi, \varphi)=W_{\varphi \phi}^{(2)}(\phi, \varphi)$.

Thus, by substituting Eqs. (34) and (35) in Eq. (37), we get the following constraint

$$
\begin{aligned}
0= & p_{1} g_{\varphi}(\varphi)+p_{2} g_{\varphi}(\phi, \varphi)-q_{2} \tilde{g}_{\phi}(\phi, \varphi)-q_{3} \tilde{g}_{\phi}(\phi) \\
& +a_{1} W_{\phi \varphi}^{(1)}(\varphi)+a_{2} W_{\phi \varphi}^{(1)}(\phi, \varphi)-b_{2} \widetilde{W}_{\varphi \phi}^{(1)}(\phi, \varphi) \\
& -b_{3} \widetilde{W}_{\varphi \phi}^{(1)}(\phi) .
\end{aligned}
$$

The above constraint allows us to obtain the specific form of the functions $g$ and $\tilde{g}$. After doing that, we can go back to the system given by Eqs. (34) and (35), and perform simple integrations to finally determine the form of $W^{(2)}(\phi, \varphi)$. In what follows, we will illustrate the extension method by explicitly constructing new interesting two-scalar fields models.

4.1 $\phi^{4}$ model coupled with $\varphi^{6}$-like model

Now, we will consider a model constructed through the coupling of the standard $\phi^{4}$ model and the $\varphi^{6}$-like model [41]. Let us start from Eq. (18), namely

$W_{\phi}^{(1)}(\phi)=\alpha\left(1-\phi^{2}\right)$,

with the deformation function given in (20), namely,

$\phi=f(\varphi)=|\varphi|-1$.

The deformed model is the $\varphi^{6}$-like model, whose superpotential satisfies Eq. (22),

$\widetilde{W}_{\varphi}^{(1)}(\varphi)=\alpha \varphi(2-|\varphi|)$.

Now, we will use the deformation function to write Eq. (39) in three equivalent arbitrary forms, where one of these must be a function of only $\phi$, another function of $\phi$ and $\varphi$, and the last function only $\varphi$, that is

$$
\begin{aligned}
W_{\phi}^{(1)}(\varphi) & =\alpha|\varphi|(2-|\varphi|), \\
W_{\phi}^{(1)}(\phi, \varphi) & =\alpha(1+\phi(1-|\varphi|)), \\
W_{\phi}^{(1)}(\phi) & =\alpha\left(1-\phi^{2}\right) .
\end{aligned}
$$

Similarly, we can use the inverse deformation function to write Eq. (41) in the following three different forms,

$$
\begin{aligned}
\widetilde{W}_{\varphi}^{(1)}(\varphi) & =\alpha \varphi(2-|\varphi|), \\
\widetilde{W}_{\varphi}^{(1)}(\phi, \varphi) & =\alpha \varphi(1-\phi), \\
\widetilde{W}_{\varphi}^{(1)}(\phi) & =\alpha \epsilon\left(1-\phi^{2}\right),
\end{aligned}
$$

where the constant parameter $\epsilon= \pm 1$ for solutions $\varphi_{ \pm}$(23), respectively. Now, by substituting these expressions directly into the constraint (38), we get

$$
g(\phi, \varphi)=-\frac{\alpha}{p_{2}}\left(\frac{b_{2}}{2} \varphi^{2}+2 b_{3} \phi|\varphi|\right),
$$


$\tilde{g}(\phi, \varphi)=\frac{\epsilon \alpha}{q_{2}}\left(2 a_{1} \phi(1-|\varphi|)-\frac{a_{2}}{2} \phi^{2}\right)$,

where we have chosen $p_{1}=0$ and $q_{3}=0$, so $p_{3}=-p_{2}$ and $q_{1}=-q_{2}$, for simplicity. In addition, we can use the deformation function, and its inverse, to write

$$
\begin{aligned}
& p_{3} g(\phi)=\frac{\alpha b_{2}}{2}(1+\phi)^{2}+2 \alpha b_{3} \phi(1+\phi), \\
& q_{1} \tilde{g}(\varphi)=\epsilon \alpha\left(2 a_{1}+\frac{a_{2}}{2}\right)(1-|\varphi|)^{2} .
\end{aligned}
$$

By substituting the above results in Eqs. (34) and (35), we obtain respectively,

$$
\begin{aligned}
W_{\phi}^{(2)}(\phi, \varphi)=\alpha & {\left[a_{1}|\varphi|(2-|\varphi|)+a_{2}(1+\phi(1-|\varphi|))\right.} \\
& -\frac{b_{2}}{2} \varphi^{2}-2 b_{3} \phi|\varphi|+a_{3}\left(1-\phi^{2}\right) \\
& \left.+\frac{b_{2}}{2}(1+\varphi)^{2}+2 b_{3} \varphi(1+\varphi)\right] \\
W_{\varphi}^{(2)}(\phi, \varphi)= & \alpha\left[b_{1} \varphi(2-|\varphi|)+\epsilon\left(2 a_{1}+\frac{a_{2}}{2}\right)(1-|\varphi|)^{2}\right. \\
& +b_{2} \varphi(1-\phi)+2 a_{1} \epsilon \phi(1-|\varphi|) \\
& \left.-\epsilon\left(b_{3}+\frac{a_{2}}{2}\right) \phi^{2}+\epsilon b_{3}\right],
\end{aligned}
$$

which upon being integrated results in the following superpotential,

$$
\begin{aligned}
W^{(2)}(\phi, \varphi)= & \alpha\left[\frac{1}{2}(a-b+c+1)|\varphi|-\frac{1}{2}(a+c-1) \varphi^{2}\right. \\
& +\frac{1}{6}(a+b+c-1)|\varphi| \varphi^{2}-\frac{1}{2}(a+b) \phi \varphi^{2} \\
& +\frac{1}{2}(2-a+b) \phi+\frac{1}{2}(1+c-a) \phi^{2} \\
& -\frac{1}{6}(a+b-2 c) \phi^{3}-\frac{1}{6}(a-2 b+c-1) \\
& \left.+\frac{1}{2}(a+b-c-1) \phi^{2}|\varphi|+a \phi|\varphi|\right], \quad \text { (48) }
\end{aligned}
$$

where we have just renamed the parameters: $a \equiv 2 a_{1}, b \equiv$ $b_{2}$, and $c \equiv 2 a_{1}+a_{2}-b_{2}-2 b_{1}+1$. This superpotential describes the coupling between the $\phi^{4}$ model and the $\varphi^{6}$ like model, and therefore from now on we will name it as the extended $\left(\phi^{4}+\varphi_{l}^{6}\right)$ model. Note that there are several models that can be considered depending on the choice of these parameters. This model contains three minima at the following values $m_{1}=(-1,0), m_{2}=(1,2)$, and $m_{3}=$ $(1,-2)$. It supports then three topological sectors, with only two of them are BPS, they are: the sector connecting $m_{1}$ and $m_{2}$, and the one connecting $m_{1}$ to $m_{3}$, with the explicit solutions given by Eqs. (19) and (23), namely

$\phi(x)=\tanh (\alpha x), \quad \varphi_{ \pm}(x)= \pm(1+\tanh (\alpha x))$,

which energy is $E_{\mathrm{BPS}}=8 \alpha / 3$. On the other hand, the nonBPS configuration, connecting the minima $m_{2}$ and $m_{3}$, do not satisfy the first-order equation. In this case, we can write an explicit solution for the specific values $a=1, b=-1$, and $c=0$,

$\phi=1, \quad \varphi_{ \pm}(x)= \pm 2 \tanh (\alpha x)$,

with energy $E=16 \alpha / 3$, which is twice the energy of the BPS sectors, as it was already expected. It is worth noting that the corresponding anti-kink configurations,

$\phi(x)=-\tanh (\alpha x), \quad \varphi_{ \pm}^{(-)}(x)= \pm(1-\tanh (\alpha x))$,

are also in the BPS sectors connecting $m_{2}$ and $m_{3}$ minima to $m_{1}$, respectively. There are several others topological sectors that appear after choosing the values of the parameters. For instance, for $a=b=0$ and $c=-1$, we recovery the BPS sector associated with the $\phi^{4}$ model, connecting the two minima $( \pm 1,0)$, with energy $E_{\mathrm{BPS}}=4 \alpha / 3$. On the other hand, we can also verify that the trivial configuration $\phi=0$ does not belong to the minima space of this potential. Finally, we would like to pointing out that for the values $a=c=1$, and $b=0$, the superpotential $W^{(2)}(\phi, \varphi)$ becomes harmonic, and consequently all the solution will be BPS solutions [46, 47].

\section{$4.2 \phi^{4}$ model coupled to sine-Gordon model}

Our starting point will be again the $\phi^{4}$ model. Now, by using the deformation function (24) we will write the right-hand side of Eq. (18) in the following forms,

$$
\begin{aligned}
W_{\phi}^{(1)}(\phi) & =\alpha\left(1-\phi^{2}\right), \\
W_{\phi}^{(1)}(\chi) & =\alpha \cos ^{2}(\beta \chi), \\
W_{\phi}^{(1)}(\phi, \chi) & =\alpha(1-\phi \sin (\beta \chi)),
\end{aligned}
$$

and similarly Eq. (25) as

$$
\begin{aligned}
\widetilde{W}_{\chi}^{(1)}(\chi) & =\frac{\alpha}{\beta} \cos (\beta \chi), \\
\widetilde{W}_{\chi}^{(1)}(\phi) & =\frac{\alpha}{\beta} \sqrt{1-\phi^{2}}, \\
\widetilde{W}_{\chi}^{(1)}(\phi, \chi) & =\frac{\alpha}{\beta} \sqrt{1-\phi \sin (\beta \chi)} .
\end{aligned}
$$

We see that the last two forms for $\widetilde{W}_{\chi}^{(1)}$ contain square root functionals, and then it is convenient to consider the following choice of parameters $b_{2}=b_{3}=0$. Also, without loss generality we choose $p_{1}=p_{2}=q_{3}=0$. This implies immediately that $b_{1}=1, p_{3}=0$, and $q_{2}=-q_{1}$. Then, by solving the constraint (38) to determine the $\tilde{g}$-function, we get

$\tilde{g}(\phi, \chi)=-\left(\frac{\alpha \beta}{q_{2}}\right)\left(2 a_{1} \phi \sin (\beta \chi)+\frac{a_{2}}{2} \phi^{2}\right) \cos (\beta \chi)$, 
which can be rewritten, by using the deformation function, as follows

$\tilde{g}(\chi)=\left(\frac{\alpha \beta}{q_{1}}\right)\left(2 a_{1}+\frac{a_{2}}{2}\right) \sin ^{2}(\beta \chi) \cos (\beta \chi)$.

By substituting the above results in Eqs. (34) and (35), we get

$$
\begin{aligned}
W_{\phi}^{(2)}(\phi, \chi)= & \alpha\left[a_{1} \cos ^{2}(\beta \chi)+a_{2}(1-\phi \sin (\beta \chi))\right. \\
& \left.+\left(1-a_{1}-a_{2}\right)\left(1-\phi^{2}\right)\right], \\
W_{\chi}^{(2)}(\phi, \chi)= & \alpha\left[\frac{1}{\beta} \cos (\beta \chi)-a_{1} \beta \phi \sin (2 \beta \chi)\right. \\
& +\beta\left(2 a_{1}+\frac{a_{2}}{2}\right) \sin ^{2}(\beta \chi) \cos (\beta \chi) \\
& \left.-\frac{a_{2} \beta}{2} \phi^{2} \cos (\beta \chi)\right] .
\end{aligned}
$$

Integrating out these expressions we finally obtain the twofields superpotential, which is given by the following form

$$
\begin{aligned}
W^{(2)}(\phi, \chi)= & \alpha\left[\phi-\left(1-a_{1}-a_{2}\right) \frac{\phi^{3}}{3}-a_{1} \phi \sin ^{2}(\beta \chi)\right. \\
& -\frac{a_{2}}{2} \phi^{2} \sin (\beta \chi)+\frac{1}{\beta^{2}} \sin (\beta \chi) \\
& \left.+\frac{1}{3}\left(2 a_{1}+\frac{a_{2}}{2}\right) \sin ^{3}(\beta \chi)\right] .
\end{aligned}
$$

This superpotential describes the coupling of the $\phi^{4}$ and sineGordon models, which from now on will be named as the extended $\left(\phi^{4}+\mathrm{sG}\right)$ model. It is worth pointing out that this model is a generalization of the coupled fields non-linear models found some years ago in [48].

The static kink-like solutions,

$\phi(x)=\tanh (\alpha x), \quad \chi(x)=\frac{1}{\beta} \arcsin (\tanh (\alpha x))$,

are BPS solutions of this model connecting the minima $m_{1}=$ $\left(-1,-\frac{\pi}{2 \beta}\right)$ and $m_{2}=\left(1, \frac{\pi}{2 \beta}\right)$, with BPS energy given by,

$E_{\mathrm{BPS}}=2 \alpha\left(\frac{2}{3}+\frac{1}{\beta^{2}}\right)$.

In general, we can verify that the corresponding potential possesses minima at the points $\left(-1,\left(2 k-\frac{1}{2}\right) \frac{\pi}{\beta}\right)$, and $\left(+1,\left(2 k+\frac{1}{2}\right) \frac{\pi}{\beta}\right)$, with $k \in \mathbb{Z}$. It is also worth highlighting the existence of other BPS solutions. For instance, a particular solution is

$\phi^{(-)}(x)=\frac{\left(1-a_{1}\right)-e^{\left(-2\left(1-a_{1}\right)+a_{2}\right) \alpha x}}{\left(\left(1-a_{1}\right)-a_{2}\right)+e^{\left(-2\left(1-a_{1}\right)+a_{2}\right) \alpha x}}$,

$\chi^{(-)}(x)=\frac{\pi}{\beta}\left(2 k-\frac{1}{2}\right)$,

providing that the parameters $a_{1}$ and $a_{2}$ are restricted to satisfy $a_{2}<\left(1-a_{1}\right)$, and $a_{2} \neq 2\left(1-a_{1}\right)$, otherwise $\phi^{(-)}(x)$ becomes an exponential or a constant solution, respectively. By choosing $k=0$, we notice that this solution connects the

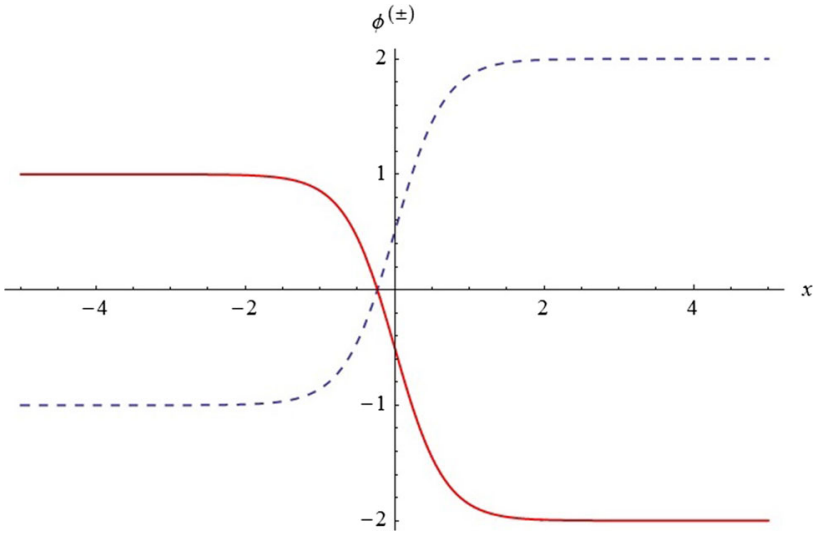

Fig. 1 The solid (red) line is the plot of the $\phi^{(+)}$solution for the parameter values $a_{1}=3$ and $a_{2}=-1$. The dashed (blue) line is the plot of the $\phi^{(-)}$solution for the parameter values $a_{1}=-1$ and $a_{2}=1$. In both cases, we have chosen $\alpha=1$, and then $E_{\mathrm{BPS}}=9 / 2$

minimum $m_{1}$ to a new minimum $m_{3}=\left(\frac{1-a_{1}}{1-a_{1}-a_{2}},-\frac{\pi}{2 \beta}\right)$. We see that the BPS energy of this solution is given by,

$E_{\mathrm{BPS}}=\frac{\alpha}{6} \frac{\left|2\left(1-a_{1}\right)-a_{2}\right|^{3}}{\left(1-a_{1}-a_{2}\right)^{2}}$.

Another possible solution is,

$\phi^{(+)}(x)=\frac{\left(1-a_{1}\right)+e^{\left(2\left(1-a_{1}\right)-a_{2}\right) \alpha x}}{\left(a_{2}-\left(1-a_{1}\right)\right)+e^{\left(2\left(1-a_{1}\right)-a_{2}\right) \alpha x}}$,

$\chi^{(+)}(x)=\frac{\pi}{\beta}\left(2 k+\frac{1}{2}\right)$.

In this case $a_{2}>\left(1-a_{1}\right)$, and again $a_{2} \neq 2\left(1-a_{1}\right)$. For $k=0$, this solution connects the minimum $m_{2}$ to a new minimum $m_{4}=\left(-\frac{1-a_{1}}{1-a_{1}-a_{2}},+\frac{\pi}{2 \beta}\right)$. We also note that the solution (63) possesses the same BPS energy as the solution (61), given by Eq. (62). We have plotted the solutions $\phi^{( \pm)}(x)$ in Fig. 1.

\subsection{E-model coupled to sine-Gordon model}

Let us consider now the sine-Gordon model and the E-model described by the first-order field equations (25) and (27), respectively, together with the deformation function (29). Then, we write the following expressions,

$$
\begin{aligned}
W_{\eta}^{(1)}(\eta) & =\alpha(1+\eta) \cos \left(\frac{1}{2} \ln (1+\eta)^{2}\right), \\
W_{\eta}^{(1)}(\chi) & =\alpha e^{\beta \chi} \cos (\beta \chi), \\
W_{\eta}^{(1)}(\eta, \chi) & =\alpha(1+\eta) \cos (\beta \chi),
\end{aligned}
$$

and

$$
\begin{aligned}
& \widetilde{W}_{\chi}^{(1)}(\chi)=\frac{\alpha}{\beta} \cos (\beta \chi), \\
& \widetilde{W}_{\chi}^{(1)}(\eta)=\frac{\alpha}{\beta} \cos \left(\frac{1}{2} \ln (1+\eta)^{2}\right),
\end{aligned}
$$


$\widetilde{W}_{\chi}^{(1)}(\eta, \chi)=\frac{2 \alpha}{\beta}\left[\cos \left(\frac{\beta \chi}{2}\right) \cos \left(\frac{1}{4} \ln (1+\eta)^{2}\right)-\frac{1}{2}\right]$.

By choosing $p_{1}=q_{3}=0$ in the constraint (38), we find the following solution,

$$
\begin{aligned}
g(\eta, \chi)= & -\alpha \frac{a_{1}}{p_{2}} e^{\beta \chi} \cos (\beta \chi) \\
& -\frac{\alpha}{\beta} \frac{b_{3}}{p_{2}} \frac{\chi}{(1+\eta)} \sin \left(\frac{1}{2} \ln (1+\eta)^{2}\right), \\
\tilde{g}(\eta, \chi)= & -\frac{2 \alpha}{\beta} \frac{b_{2}}{q_{2}} \cos \left(\frac{\beta \chi}{2}\right) \cos \left(\frac{1}{4} \ln (1+\eta)^{2}\right) \\
& -\alpha \beta \frac{a_{2}}{q_{2}}\left(\eta+\frac{\eta^{2}}{2}\right) \sin (\beta \chi) .
\end{aligned}
$$

As it was done before, we can use the deformation function to write

$$
\begin{aligned}
g(\eta)= & -\frac{\alpha a_{1}}{p_{2}}(1+\eta) \cos \left(\frac{1}{2} \ln (1+\eta)^{2}\right) \\
& -\frac{\alpha b_{3}}{\beta^{2} p_{2}} \frac{\ln (1+\eta)}{(1+\eta)} \sin \left(\frac{1}{2} \ln (1+\eta)^{2}\right) \\
\tilde{g}(\chi)= & -\frac{2 \alpha b_{2}}{\beta q_{2}} \cos ^{2}\left(\frac{\beta \chi}{2}\right)-\frac{\alpha \beta a_{2}}{2 q_{2}}\left(e^{2 \beta \chi}-1\right) \sin (\beta \chi) .
\end{aligned}
$$

Using these results, we obtain

$$
\begin{aligned}
W_{\eta}^{(2)}(\eta, \chi)= & \alpha a_{2}(1+\eta) \cos (\beta \chi) \\
& +\frac{\alpha b_{3}(\ln (1+\eta)-\beta \chi)}{\beta^{2}} \sin \left(\frac{1}{2} \ln (1+\eta)^{2}\right) \\
& +\alpha\left(1-a_{2}\right)(1+\eta) \cos \left(\frac{1}{2} \ln (1+\eta)^{2}\right)(74) \\
W_{\chi}^{(2)}(\eta, \chi)= & \frac{\alpha}{\beta}\left(1-b_{3}\right) \cos (\beta \chi) \\
& +\frac{\alpha b_{3}}{\beta} \cos \left(\frac{1}{2} \ln (1+\eta)^{2}\right) \\
& +\frac{\alpha \beta a_{2}}{2}\left(e^{2 \beta \chi}-(1+\eta)^{2}\right) \sin (\beta \chi) .
\end{aligned}
$$

We then can construct the corresponding two-fields superpotential,

$$
\begin{aligned}
W^{(2)}(\eta, \chi)= & \frac{2 \alpha}{5}\left(1-a_{2}\right)(1+\eta)^{2} \cos \left(\frac{1}{2} \ln (1+\eta)^{2}\right) \\
& +\frac{\alpha}{5}\left(1-a_{2}\right)(1+\eta)^{2} \sin \left(\frac{1}{2} \ln (1+\eta)^{2}\right) \\
& +\frac{\alpha b_{3}}{\beta^{2}}(\beta \chi-\ln (1+\eta)) \cos \left(\frac{1}{2} \ln (1+\eta)^{2}\right) \\
& +\frac{\alpha a_{2}}{10}\left[\left(5-e^{2 \beta \chi}\right) \cos (\beta \chi)+2 e^{2 \beta \chi} \sin (\beta \chi)\right] \\
& +\frac{\alpha b_{3}}{\beta^{2}} \sin \left(\frac{1}{2} \ln (1+\eta)^{2}\right)
\end{aligned}
$$

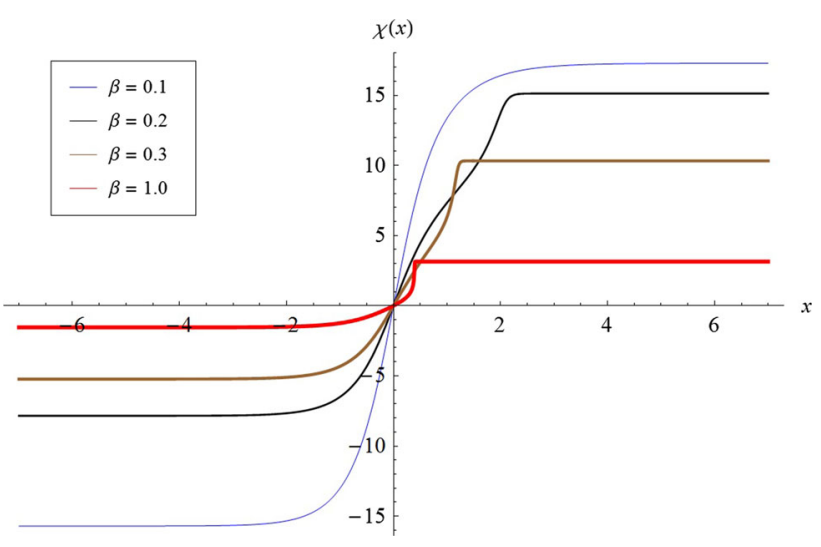

Fig. 2 Plot of the kink-like numerical solution of the Eq. (79) for $\alpha=$ 2 , and different values of $\beta$. The thickness of the curves increases as $\beta$ also increases. This solutions interpolates between the minima $\bar{\chi}_{0}$ and $\bar{\chi}_{+1}$

$$
\begin{aligned}
& +\alpha a_{2}\left(\eta+\frac{\eta^{2}}{2}\right) \cos (\beta \chi) \\
& +\frac{\alpha}{\beta^{2}}\left(1-b_{3}\right) \sin (\beta \chi) .
\end{aligned}
$$

This two-parameters superpotential leads us to a potential $V(\eta, \chi)$ describing the coupling of the sine-Gordon model and the E-model, which from now on we will named as the extended $(\mathrm{sG}+\mathrm{E})$ model. This superpotential supports the static kink-like solutions (26) and (28),

$\eta(x)=[\exp (\arctan (\sinh (\alpha x)))-1]$,

$\chi(x)=\frac{1}{\beta} \arctan (\sinh (\alpha x))$,

connecting the minima $m_{1}=\left(e^{-\pi / 2}-1,-\pi / 2 \beta\right)$ and $m_{2}=$ $\left(e^{\pi / 2}-1, \pi / 2 \beta\right)$, with BPS energy given by

$E_{\mathrm{BPS}}=\frac{2 \alpha}{5 \beta^{2}}\left(5+\beta^{2} \cosh \pi\right)$.

We notice that for the particular values of the parameters $a_{2}=$ 0 and $b_{3}=1$, it is possible to obtain other BPS solutions, at least numerically. In this case, we have that $\eta(x)=-1$, and $\chi(x)$ has to satisfy

$\chi^{\prime}(x)=\frac{\alpha}{2 \beta}\left(2 \cos (\beta \chi)+\beta^{2} e^{2 \beta \chi} \sin (\beta \chi)\right)$.

It is interesting to see that the associated potential $V(-1, \chi)$ represents a modification of the sine-Gordon model. In fact, we have verified that it has infinite minima, and supports BPS solutions. The minima are located approximately at the following points,

$\bar{\chi}_{k} \approx \begin{cases}\frac{1}{2 \beta}\left[(2 k-1) \pi+\frac{\beta^{3}}{e^{(1-2 k) \pi}-\beta^{3}}\right], & k \leq 0, \\ \frac{\pi k}{\beta}-\frac{2}{\beta^{3}} e^{-2 k \pi}, & k>0 .\end{cases}$

Then, there are at least three type of topological sectors, a small one for $k<0$, a medium one for $k=0$, and the large 
one for $k>0$. The corresponding BPS energies are given by

$$
\begin{aligned}
& E_{\mathrm{BPS}}^{(k, k+1)} \\
& \approx \begin{cases}\frac{2 \alpha}{5 \beta^{2}}\left(5+\beta^{2} e^{2 k \pi} \cosh (\pi)\right) & \\
\quad+\frac{\alpha \beta^{3}}{2}\left[\frac{\cosh (2 \pi)-\beta^{3} e^{2 \pi k} \cosh (\pi)}{\beta^{6}-2 \beta^{3} e^{-2 \pi k} \cosh (\pi)+e^{-4 \pi k}}\right], & k<0, \\
\frac{\alpha}{20}\left[\left(2 e^{2 \pi}-e^{-\pi}\right)+\frac{20}{\beta^{4}}\left(\beta^{2}+2 e^{-2 \pi}\right)\right. & \\
\left.\quad+\frac{5}{e^{\pi}-\beta^{3}}\right], & k=0, \\
\frac{\alpha \cosh (\pi)}{5 \beta^{4}}\left[20 e^{-(2 k+1) \pi}+\beta^{4} e^{(2 k+1) \pi}\right], & k>0 .\end{cases}
\end{aligned}
$$

Unfortunately, we have not been able to obtain the corresponding analytical solutions of the first-order equation (79) for the kink solutions associated to each topological sector. However, we did construct them numerically. In Fig. 2 we have plotted the numerical kink solutions connecting the minima $\bar{\chi}_{0}$ to $\bar{\chi}_{+1}$, for several values of the parameter $\beta$. We can see that for $\beta \lesssim 0.1$, the profile tends to fit the sine-Gordon kinks. For greater values it undergoes a rapid deformation. It is worth noting that this first-order approximation fails when $\beta=e^{(1-2 k) \pi / 3}$, for $k \leq 0$. However, there is nothing special about those values, but in that case a second-order approximation would be necessary.

\section{Linear stability of the BPS configurations}

Let us now discuss the linear stability for the two-scalar fields models we have constructed. The main issue is basically to analyse the spectrum of the corresponding Schrödinger-like operator associated with the normal modes of the classical model. The stability will be ensured when this Schrödingerlike is positive semi-definite, implying that negative eigenvalues will be absent from its spectrum, and the zero mode will correspond to the lowest bound state [49-52].

First of all, it is well-known for one-field models that the static configurations of the $\phi^{4}$ model (19), the $\varphi^{6}$-like model (23), the sine-Gordon model (26), and the E-model (28) are all stable $[29,41,43,53]$, with the corresponding Schrödinger-like operators related to the so-called RosenMorse II potential (or modified Pos̈chl-Teller potential) for the first three models, and to the so-called Scarf-II (hyperbolic) potential in the latter case [54] (see more details in Appendix A).

Now, the stability analysis for multi-fields models is in general a highly non-trivial problem. Here, we will follow the line of reasoning introduced in [52], to study the stability of static solutions in the two-scalar field models constructed in Sect. 4. The starting point is to consider a pair of static solutions, say $\phi_{s}(x)$ and $\varphi_{s}(x)$, and then introduce small fluctuations around these solutions, given in the following form

$$
\begin{aligned}
& \phi(x, t)=\phi_{s}(x)+\sum_{k} \rho_{k}(x) \cos \left(w_{k} t\right), \\
& \varphi(x, t)=\varphi_{s}(x)+\sum_{k} \sigma_{k}(x) \cos \left(w_{k} t\right),
\end{aligned}
$$

where $\rho_{k}$ and $\sigma_{k}$ are the small perturbations, when compared to the static configurations. Now, by substituting the fields $\phi(x, t)$ and $\varphi(x, t)$ into the second-order equations (3), and considering only first-order terms in the fluctuations, we obtain the Schrödinger-like equation $H \Psi_{k}(x)=w_{k}^{2} \Psi_{k}(x)$, where

$H=-\frac{d^{2}}{d x^{2}}+\left(\begin{array}{cc}V_{\phi \phi} & V_{\phi \varphi} \\ V_{\varphi \phi} & V_{\varphi \varphi}\end{array}\right), \quad \Psi_{k}(x)=\left(\begin{array}{c}\rho_{k}(x) \\ \sigma_{k}(x)\end{array}\right)$.

Notice that the derivatives of the potential $V(\phi, \varphi)$ are written in terms of the static fields $\phi_{s}(x)$ and $\varphi_{s}(x)$. In addition, as it can be seen from Eqs. (81) and (82), linear stability requires that the eigenvalues of $H$ have to be positive semi-definite, i.e. $w_{k}^{2} \geq 0$, with the zero mode $H \Psi_{0}(x)=0$, being given by

$\Psi_{0}(x)=N_{0}\left(\begin{array}{c}\rho_{0}(x) \\ \sigma_{0}(x)\end{array}\right)=N_{0}\left(\begin{array}{c}\phi^{\prime}(x) \\ \varphi^{\prime}(x)\end{array}\right)$,

where the normalization constant $N_{0}$ can be chosen to be the unit. When the potential $V(\phi, \varphi)$ supports BPS states the Hamiltonian in (83) can be written as follows,

$H=A_{-}^{\dagger} A_{-}=A_{+} A_{-}$,

where the first-order operators

$A_{ \pm}= \pm \frac{d}{d x}+\mathbf{W}, \quad \mathbf{W}=\left(\begin{array}{cc}W_{\phi \phi}^{(2)} & W_{\phi \varphi}^{(2)} \\ W_{\varphi \phi}^{(2)} & W_{\varphi \varphi}^{(2)}\end{array}\right)$,

have been introduced. Note that $A_{ \pm}^{\dagger}=A_{\mp}$, implies that the Schrödinger-like operator $H$ is always positive semi-definite for the BPS case, thus ensuring the linear stability of the BPS configurations. In this case, the ground state coincides with zero mode, and can be written as

$\Psi_{0}(x)=\left(\begin{array}{c}W_{\phi}^{(2)} \\ W_{\varphi}^{(2)}\end{array}\right)$.

Here, we will have an inherent difficulty regarding the explicit determination of the eigenvalue spectrum of the associated Schrödinger-like operator. As it can be seen, the coupling between static fields results in the coupling of the fluctuations in (83). However, the problem turns to be more manageable if we take advantage of the first-order operators (86), and diagonalize the matrix $\mathbf{W}$, to obtain

$A_{ \pm}= \pm \frac{d}{d x}+\left(\begin{array}{cc}u_{+} & 0 \\ 0 & u_{-}\end{array}\right)$ 
where the respective eigenvalues are [52]

$$
\begin{aligned}
u_{ \pm}= & \frac{1}{2}\left(W_{\phi \phi}^{(2)}+W_{\varphi \varphi}^{(2)}\right) \\
& \pm \sqrt{\frac{1}{4}\left(W_{\phi \phi}^{(2)}-W_{\varphi \varphi}^{(2)}\right)^{2}+\left(W_{\phi \varphi}^{(2)}\right)^{2}} .
\end{aligned}
$$

By substituting (88) in (85), we obtain two decoupled eigenvalue equations

$$
\begin{aligned}
& {\left[-\frac{d^{2}}{d x^{2}}+U_{+}(x)\right] \rho_{k}(x)=w_{k}^{2} \rho_{k}(x),} \\
& {\left[-\frac{d^{2}}{d x^{2}}+U_{-}(x)\right] \sigma_{k}(x)=w_{k}^{2} \sigma_{k}(x),}
\end{aligned}
$$

where the quantum mechanical potentials are given by

$U_{ \pm}(x)=u_{ \pm}^{2}+\frac{d u_{ \pm}}{d x}$.

It is worth pointing out that in general this method would require certain simplifications since the square root term appearing in Eq. (89) brings some complications for the explicit analytical calculations. In what follows, we will try then to simplify this term whenever is possible to perform the analytical analysis of the stability of the BPS configurations for the two-fields models we have constructed. Otherwise, the corresponding spectral problems should be analysed from a numerical point of view.

\subsection{The extended $\left(\phi^{4}+\varphi_{l}^{6}\right)$ model}

Let us first study the stability of the BPS solutions (49) of the extended $\left(\phi^{4}+\varphi_{l}^{6}\right)$ model. From the superpotential (48), and the BPS solutions Eq. (49), we obtain

$u_{+}=2 b+2 c \tanh (x)$,

$u_{-}=-2 \tanh (x)$,

where we are assuming that $\alpha=1$, and

$b \geq|1+c|$,

in order to simplify the square root term. Using these results, we get the corresponding quantum mechanical potentials (see Fig. 3),

$$
\begin{aligned}
U_{-}(x)= & 4-6 \operatorname{sech}^{2}(x) \\
U_{+}(x)= & 4\left(b^{2}+c^{2}\right)+8 b c \tanh (x) \\
& -2 c(2 c-1) \operatorname{sech}^{2}(x),
\end{aligned}
$$

They have again the form of the Rosen-Morse II potentials [54] (see Appendix A). In this case the parameters of the potential $U_{+}(x)(97)$ are given by,

$A=-2 c, \quad B=4 b c$.
Then, from the stability condition (A.3), we see that the parameters have to satisfy

$c<0, \quad|b|<|c|, \quad 0 \leq k<A-\sqrt{4|b||c|}$.

Now, let us choose some interesting values for the parameters. From Eq. (95), we note that if $b=0$, then $c=-1$, and we get that

$U_{+}(x)=U_{-}(x)=4-6 \operatorname{sech}^{2}(x)$,

both potentials are equal, and stability can be guaranteed. On the other hand, when $b<0$ the condition (95) is not satisfied, and then stability cannot be proven in that case, at least analytically. Finally, by considering $b>0$, together with the conditions (95) and (99), we find that

$|1+c| \leq b<|c|$,

and then $c<-1 / 2$ for consistency. Furthermore, analysing possible values of number of bound states $k$, we see that if $-\frac{1}{2}\left(1+\frac{\sqrt{2}}{2}\right)<c<-\frac{1}{2}$, we will have only the zero mode $k=0$. For values $c \leq-\frac{1}{2}\left(1+\frac{\sqrt{2}}{2}\right)$, we have the following possibilities,

$k= \begin{cases}0, & \text { if } \frac{1}{|c|}\left(c+\frac{1}{2}\right)^{2} \leq b<|c|, \\ 0 \text { and 1, } & \text { if }|1+c| \leq b<\frac{1}{|c|}\left(c+\frac{1}{2}\right)^{2} .\end{cases}$

We can also see that, since the potential $U_{-}$has eigenvalues $E_{0}=0$ and $E_{1}=3$, it will have common eigenvalues with $U_{+}$only if

$b=\sqrt{\frac{(1+c)(1+2 c)^{2}}{(1+4 c)}}, \quad\left(-1-\frac{\sqrt{3}}{2}\right)<c<-1$.

In the Table 1, we have chosen some particular values for the parameters in order to illustrate our results. For all these cases, the stability of the solutions is guaranteed.

\subsection{The extended $\left(\phi^{4}+\mathrm{sG}\right)$ model}

Now, we will analyze the stability of the BPS solutions (59) of the extended $\left(\phi^{4}+\mathrm{sG}\right)$ model described by the superpotential (58). For sake of simplification, we have chosen $a_{2}=-2 a_{1}$, and $\alpha=1$, to get

$u_{ \pm}=-\frac{3}{2} \tanh (x) \pm \frac{1}{2} \operatorname{sgn}(x) \tanh (x)$,

where $\operatorname{sgn}(x)$ is the signum function. Then, we obtain the following quantum-mechanical potentials,

$U_{ \pm}(x)=\frac{1}{2}(5 \mp 3 \operatorname{sgn}(x))-2(2 \mp \operatorname{sgn}(x)) \operatorname{sech}^{2}(x)$.

In general, they are also associated to the Rosen-Morse II potential (A.1), with $B=0$ and $\alpha=1$. However, these quantum-mechanical potentials are discontinuous, as it can 


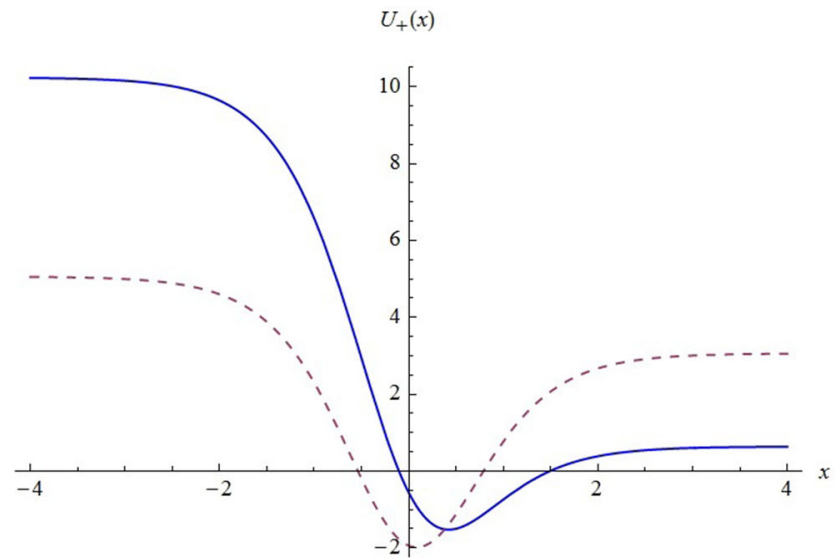

Fig. 3 Quantum mechanical potential $U_{+}(x)$ associated to the extended $\left(\phi^{4}+\varphi_{l}^{6}\right)$ model for different values of the parameters. On the left, we have plotted the potential with $c=-1$, and $b=0.6$ (solid

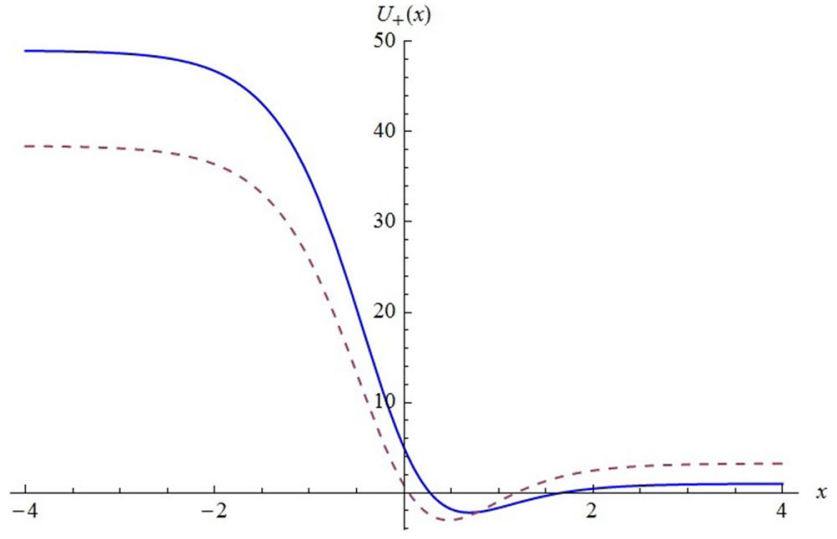

line), and $b=0.125$ (dashed line). On the right, we have plotted the potential with $c=-2$, and $b=1.5$ (solid line), and $b=1.1$ (dashed line)

Table 1 Number of bound states and their eigenvalues for different values of the parameters $b$ and $c$

\begin{tabular}{llll}
\hline$c$ & $b$ & $k$ & $E_{k}=\omega_{k}^{2}$ \\
\hline-1 & 0.6 & 0 & 0 \\
-1 & 0.125 & 0 & 0 \\
-2 & & 1 & 2.81 \\
-2 & 1.5 & 0 & 0 \\
& 1.1 & 0 & 0 \\
\hline
\end{tabular}
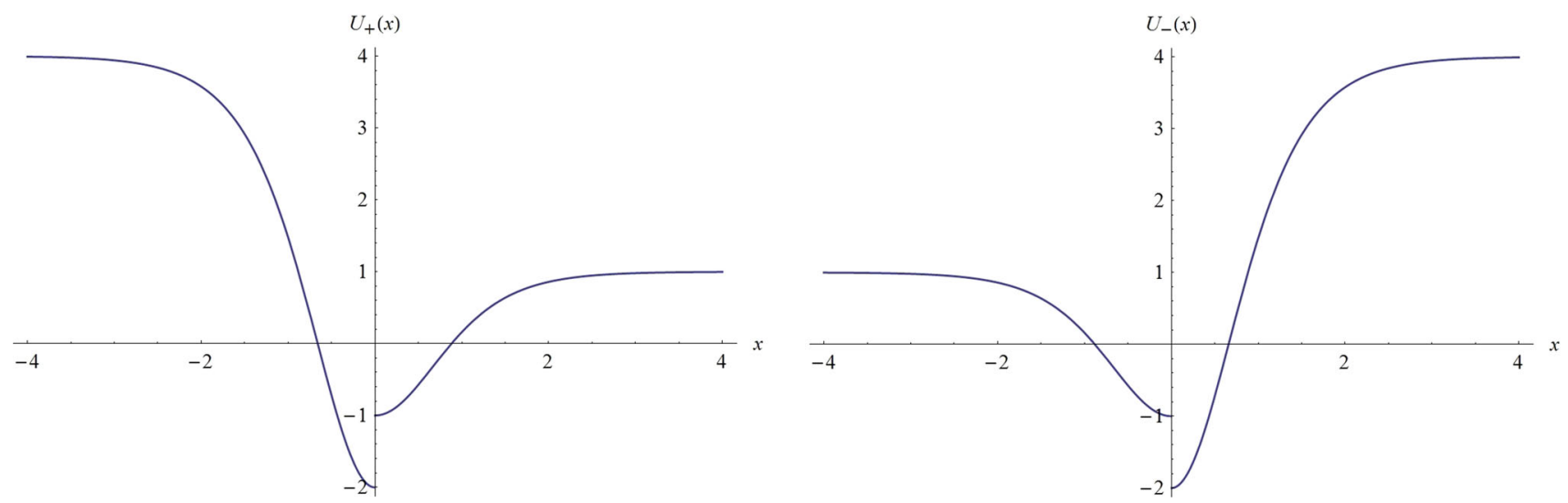

Fig. 4 Discontinuous quantum-mechanical potentials $U_{ \pm}(x)$ associated to the extended $\left(\phi^{4}+\mathrm{sG}\right)$ model, for $\alpha=1$ and $a_{2}=-2 a_{1}$

be seen from Fig. 4, and that novel feature will require special attention in order to determine the eigenvalues. To do that, we will use the procedure introduced in $[55,56]$ to determine the energy levels of composite potentials, which is based on the so-called Green function factorization theorem [57]. The main idea consists in decomposing the discontinuous potential into two "pieces", namely

$U(x)=U^{(\mathrm{L})}(x) \theta(-x)+U^{(\mathrm{R})}(x) \theta(x)$, where $\theta(x)$ is the unit step function, and $U^{(\mathrm{L} / \mathrm{R})}(x)$ are continuous and symmetric (around the origin) potentials, for which the corresponding energy levels and wave functions for all stationary states are assumed to be known. Then, by considering the Green functions $G^{(\mathrm{L} / \mathrm{R})}\left(x, x^{\prime} ; E\right)$ associated to each potentials $U^{(\mathrm{L})}$ and $U^{(\mathrm{R})}$, the allowed eigenvalues $E$ of the composite potential $U$ will be given by the solutions 
of the following transcendental equation $[55,56]$,

$G^{(\mathrm{L})}(0,0 ; E)+G^{(\mathrm{R})}(0,0 ; E)=0$.

In our case, both Green functions are associated to the solvable Rosen-Morse II potential, and its explicit formula can be written as follows [58],

$$
\begin{aligned}
G\left(x, x^{\prime} ; E\right) & \\
= & -\frac{i}{2} \Gamma\left(\sqrt{A^{2}-E}-A\right) \Gamma\left(1+A+\sqrt{A^{2}-E}\right) \\
& \times\left[\theta\left(x-x^{\prime}\right) P_{A}^{-\sqrt{A^{2}-E}}(\tanh (x)) P_{A}^{-\sqrt{A^{2}-E}}\left(-\tanh \left(x^{\prime}\right)\right)\right. \\
& \left.+\left(x \leftrightarrow x^{\prime}\right)\right],
\end{aligned}
$$

where $A$ is the parameter given in (A.1), $\Gamma(z)$ is the Gamma function, and

$P_{a}^{b}(z)=\left(\frac{1+z}{1-z}\right)^{\frac{b}{2}} \frac{1}{\Gamma(1-b)} F\left(-a, 1+a ; 1-b ; \frac{1-z}{2}\right)$,

are the associated Legendre polynomials, which are defined in terms of the hypergeometric function $F(a, b ; c ; z)$. Therefore, for the $U_{+}(x)$ potential, we have that

$U_{+}^{(\mathrm{L})}=4-6 \operatorname{sech}^{2}(x), \quad U_{+}^{(\mathrm{R})}=1-2 \operatorname{sech}^{2}(x)$,

and then

$G_{+}^{(\mathrm{L})}(0,0 ; E)=\frac{i}{2} \frac{(3-E)}{E \sqrt{4-E}}$,

$G_{+}^{(\mathrm{R})}(0,0 ; E)=\frac{i}{2} \frac{\sqrt{1-E}}{E}$.

From these results, we see that the transcendental equation (107) becomes

$E \sqrt{1-E} \sqrt{4-E}+E(3-E)=0$,

which only allows $E=0$ in the spectrum of the discontinuous potential $U_{+}(x)$. We can see that the zero energy eigenvalue is common to the decomposed potentials, which is consistent with the fact that $E=0$ is a pole of the Green function (108) for both cases. An identical transcendental equation will be obtained for the potential $U_{-}(x)$, since $U_{-}^{(\mathrm{L} / \mathrm{R})}=U_{+}^{(\mathrm{R} / \mathrm{L})}$, which again will allow only the zero energy eigenvalue. These results lead us to ensure the stability for the BPS solutions of the extended $\left(\varphi^{4}+\mathrm{sG}\right)$ model, at least for our particular choice of parameters.

\subsection{The extended $(\mathrm{sG}+\mathrm{E})$ model}

Finally, let us study the stability of the BPS solutions (77) of the extended $(\mathrm{sG}+\mathrm{E})$ superpotential (76). In this case, we find that

$u_{ \pm}(x)=\frac{\alpha}{2}\left\{\operatorname{sech}(\alpha x)+\left(a_{2}+b_{3}-2\right) \tanh (\alpha x)\right.$

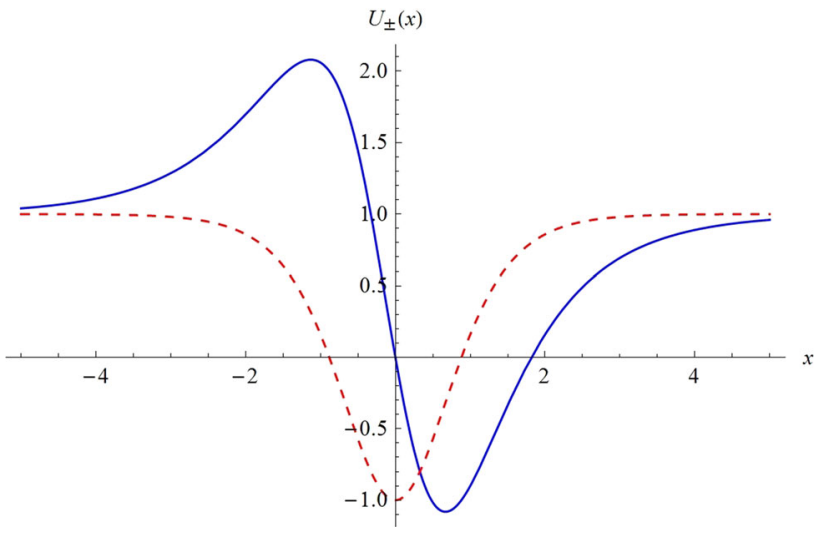

Fig. 5 Quantum-mechanical potentials $U_{+}$(dashed line) and $U_{-}$(solid line) of the extended ( $\mathrm{sG}+\mathrm{E})$ model for $a_{2}=b_{3}=0$, and $\alpha=1$

$$
\begin{aligned}
& +\left(\frac{b_{3}}{\beta^{2}} e^{-2 \operatorname{gd}(x)}+a_{2} \beta^{2} e^{2 \operatorname{gd}(x)}\right) \tanh (\alpha x) \\
& \pm 2\left[\left(\frac{b_{3}}{\beta} e^{-\operatorname{gd}(x)}+a_{2} \beta e^{\operatorname{gd}(x)}\right)^{2} \tanh ^{2}(\alpha x)\right. \\
& +\frac{e^{-4 \operatorname{gd}(x)}}{4 \beta^{4}}\left(\beta^{2} e^{2 \operatorname{gd}(x)} \operatorname{sech}(\alpha x)\right. \\
& -\left(\beta^{2} e^{2 \operatorname{gd}(x)}-1\right)\left(b_{3}+a_{2} \beta^{2} e^{2 \operatorname{gd}(x)}\right) \\
& \left.\left.\times \tanh (\alpha x))^{2}\right]^{\frac{1}{2}}\right\}
\end{aligned}
$$

where $\operatorname{gd}(x)=\arctan (\sinh x)$, is the Gudermannian function. In order to simplify the root term in Eq. (114), and study analytically the associated quantum-mechanical potentials, we could choose $a_{2}=b_{3}=0$, obtaining

$$
\begin{aligned}
& U_{-}^{(0)}(x)=\alpha^{2}-2 \alpha^{2} \operatorname{sech}^{2}(\alpha x), \\
& U_{+}^{(0)}(x)=\alpha^{2}-\alpha^{2} \operatorname{sech}^{2}(\alpha x)-3 \alpha^{2} \operatorname{sech}(\alpha x) \tanh (\alpha x),
\end{aligned}
$$

which correspond to the Scarf II (A.6) and Rosen-Morse II (A.1) potentials, respectively. However, this choice of parameters trivially decouples the fields $\eta$ and $\chi$. See their plots in Fig. 5. From the analytical point of view it is quite complicated to study these quantum-mechanical potentials for general values of the parameters $a_{2}$ and $b_{3}$. Instead, we will perform a more qualitative and approximated analysis of the bound states for some values of the parameters. At this point, we can only guarantee that stability does exist at least for some very small values of the parameters, that is, the potentials possess the zero-mode as their fundamental bound state, and there is no negative energy eigenvalues. Of course, a more precise analysis requires a deeper numerical study.

Before doing that, let us take a look of the potential deformations for some small values of the parameters. In the Figs. 6 and 7 we have plotted the potentials for some configurations with $a_{2}=0$, and small values of $b_{3}$. While, in Figs. 8 

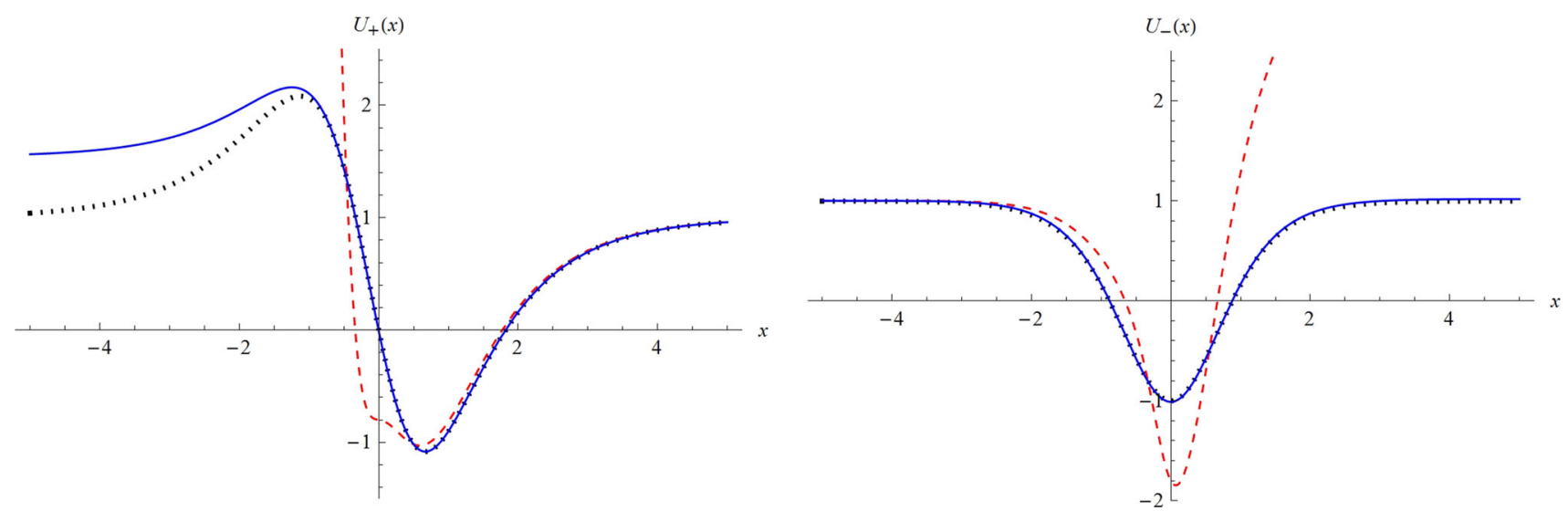

Fig. 6 Quantum-mechanical potentials $U_{+}$(on the left) and $U_{-}$(on the right) for $a_{2}=0$ and $\alpha=1$. For both, we have plotted the values $b_{3}=0$, $b_{3}=-0.01$, and $b_{3}=-0.8$, depicted with dotted, solid, and dashed lines, respectively
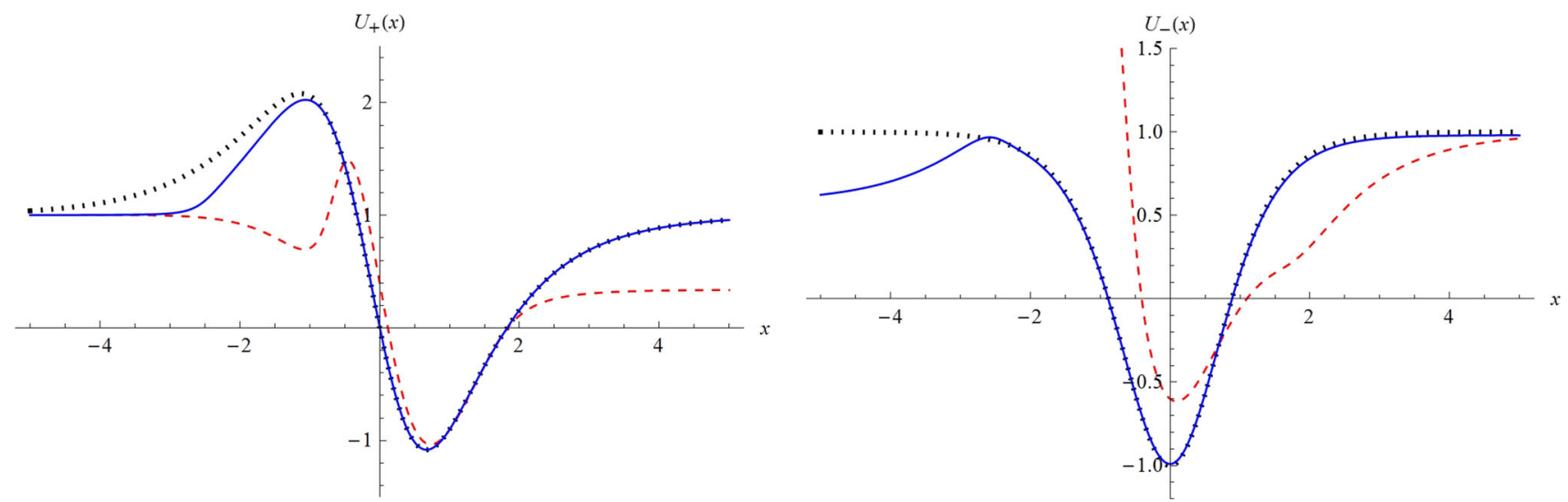

Fig. 7 Quantum-mechanical potentials $U_{+}$(on the left) and $U_{-}$(on the right) for $a_{2}=0$ and $\alpha=1$. For both, we have plotted the values $b_{3}=0$, $b_{3}=0.01$, and $b_{3}=0.4$, depicted with dotted, solid, and dashed lines, respectively

and 9 , we have plotted configurations with $b_{3}=0$, and small values of $a_{2}$. Now, for very small values of the parameters $\left(\lesssim 10^{-2}\right)$, it is clear that the quantum-mechanical potentials converge to exactly solvable problem, see also Fig. 10.

In that case it is possible to apply the time-independent perturbation theory for the calculation of the energy eigenvalues corrections. To do that let us first consider the case when $a_{2}=0$ and $b_{3}=\lambda \lesssim 10^{-2}$, so we have a perturbed Hamiltonian which consists of two parts

$H=H_{0}+\lambda H_{1}$,

with

$$
\begin{aligned}
& H_{0}=-\frac{d^{2}}{d x^{2}}+\left(\begin{array}{cc}
U_{+}^{(0)} & 0 \\
0 & U_{-}^{(0)}
\end{array}\right), \\
& H_{1}=\left(\begin{array}{cc}
U_{+}^{(1)} & 0 \\
0 & U_{-}^{(1)}
\end{array}\right),
\end{aligned}
$$

where $U_{ \pm}^{(0)}$ are the exactly solvable potentials (116) and (116), and the first-order corrections $U_{ \pm}^{(1)}$ are given in this case by

$U_{+}^{(1)}=-\frac{\alpha^{2}}{\beta^{2}} e^{-2 \arctan (\sinh (\alpha x))}\left(2-3 \operatorname{sech}^{2}(\alpha x)\right)$,

$U_{-}^{(1)}=-\alpha^{2}\left(2-3 \operatorname{sech}^{2}(\alpha x)\right)$.

Then, the eigenvalues $E_{k}$ of the perturbed problem can be expanded in a power series in the parameter $\lambda$ as follows

$E_{k}=E_{k}^{(0)}+\lambda E_{k}^{(1)}+\cdots$,

where $E_{k}^{(0)}$ are the unperturbed eigenvalues given in (A.2) and (A.7), respectively, and the first-order correction $E_{k}^{(1)}$ can be obtained from the expression

$$
E_{k}^{(1)}=\int_{-\infty}^{\infty} d x\left[\rho_{k}^{*}(x) U_{+}^{(1)}(x) \rho_{k}(x)+\sigma_{k}^{*}(x) U_{-}^{(1)}(x) \sigma_{k}(x)\right],
$$

where $\rho_{k}(x)$ and $\sigma_{k}(x)$ will be given in this case by the Scarf II (A.8) and Rosen-Morse II (A.4) eigenfunctions, respectively. In fact, from the explicit form of the potentials (116) and (116), we see that the associated parameters are $A=\alpha$ in 

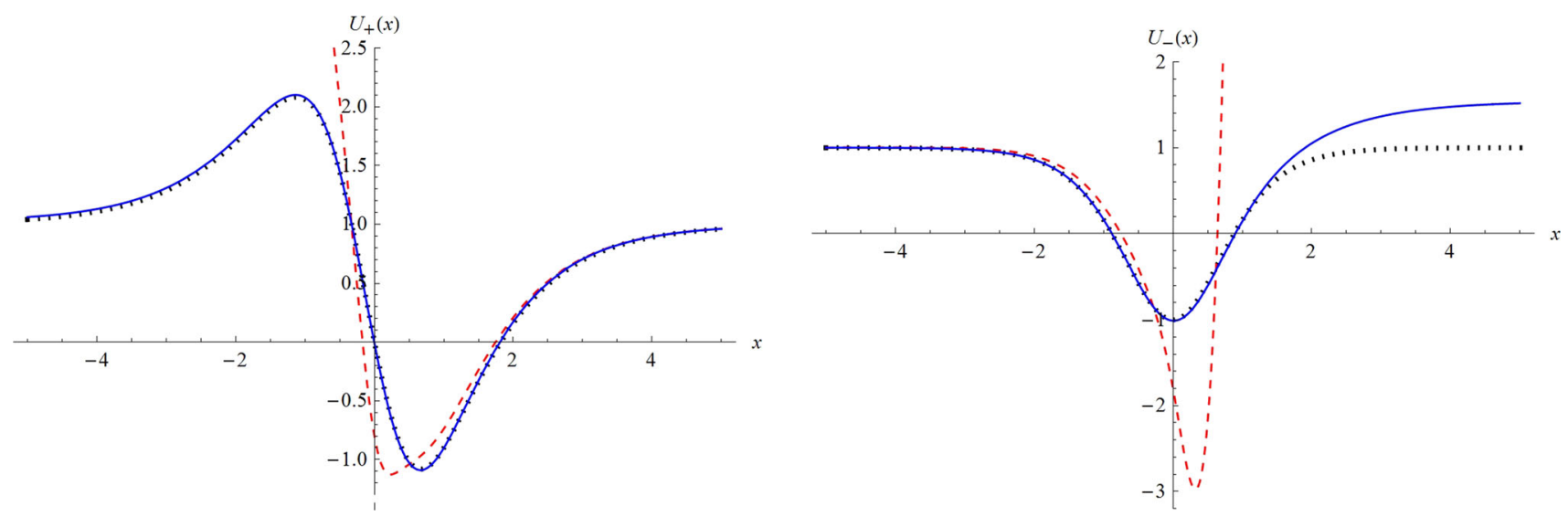

Fig. 8 Quantum-mechanical potentials $U_{+}$(on the left) and $U_{-}$(on the right) for $b_{3}=0$ and $\alpha=1$. For both, we have plotted the values $a_{2}=0$, $a_{2}=-0.01$, and $a_{2}=-0.8$, depicted with dotted, solid, and dashed lines, respectively
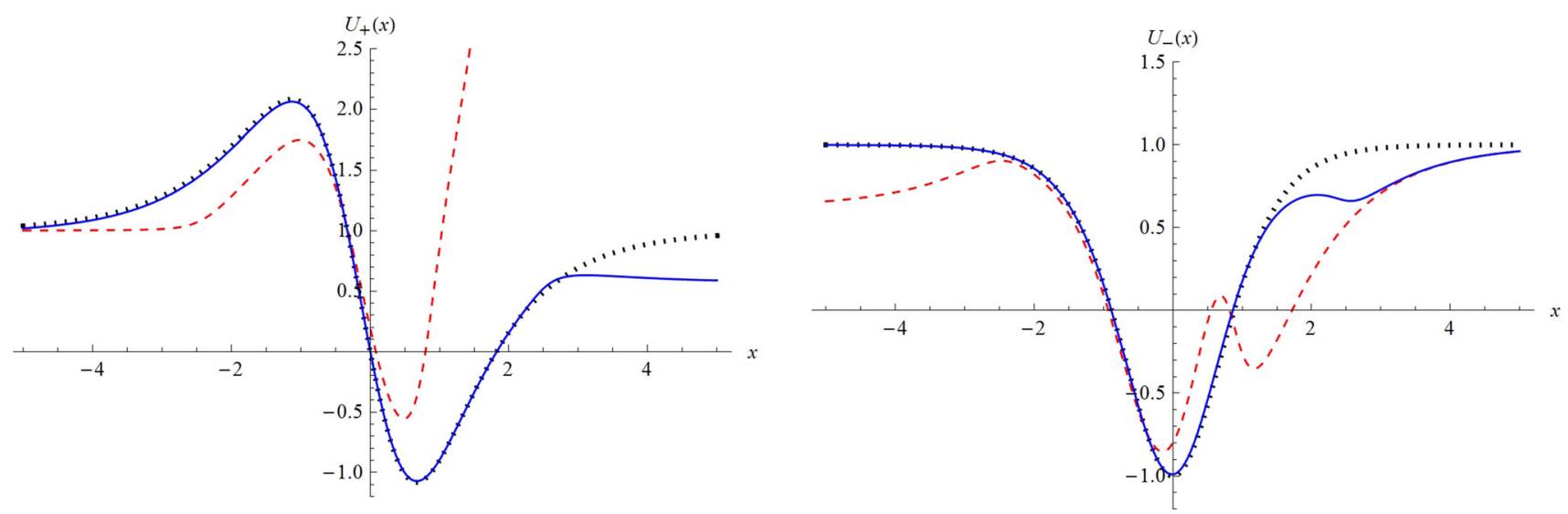

Fig. 9 Quantum-mechanical potentials $U_{+}$(on the left) and $U_{-}$(on the right) for $b_{3}=0$ and $\alpha=1$. For both, we have plotted the values $a_{2}=0$, $a_{2}=0.01$, and $a_{2}=0.2$, depicted with dotted, solid, and dashed lines, respectively
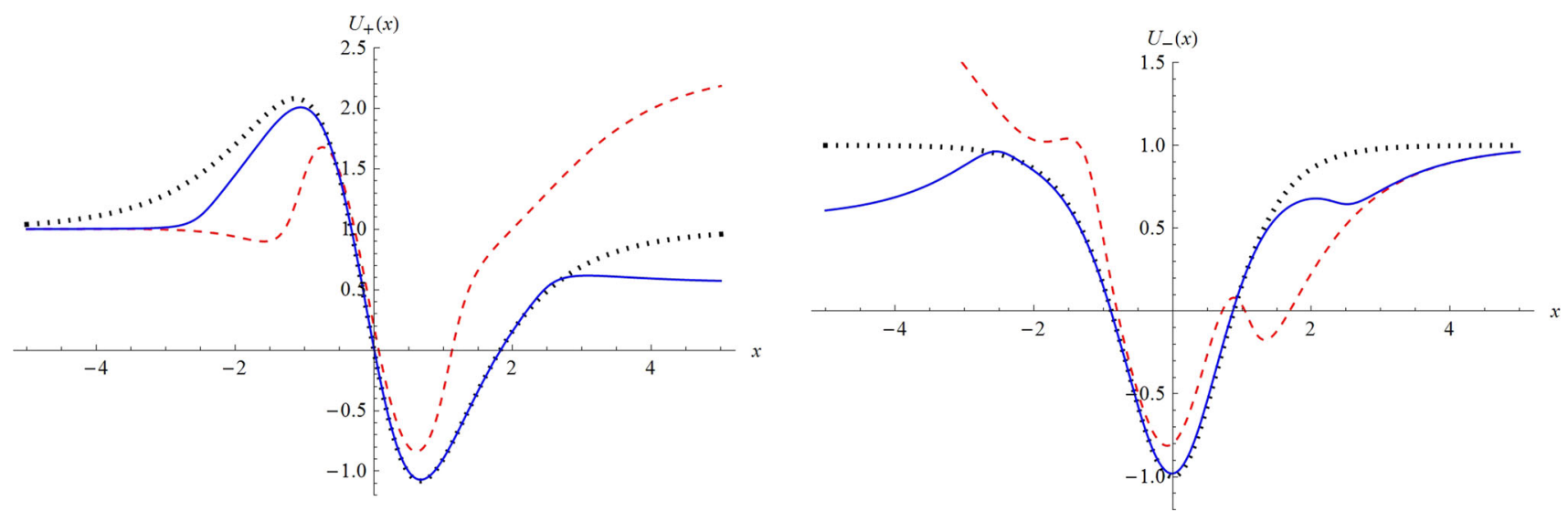

Fig. 10 Quantum-mechanical potentials $U_{+}$(on the left) and $U_{-}$(on the right) for $a_{2}=0$ and $b_{3}=0$ (dotted line), $a_{2}=0.01$ and $b_{3}=0.01$ (solid line), and $a_{2}=0.1$ and $b_{3}=0.1$ (dashed line) 
both cases, and $B=-\alpha$ for $U_{+}^{(0)}$, and $B=0$ for $U_{-}^{(0)}$. Thus, both potentials possess only one bound state, the zero mode,

$\Psi_{0}=\left(\begin{array}{c}\rho_{0}(x) \\ \sigma_{0}(x)\end{array}\right)=\left(\begin{array}{c}\operatorname{sech}(\alpha x) e^{\operatorname{gd}(x)} \\ \operatorname{sech}(\alpha x)\end{array}\right)$,

with energy $E_{0}^{(0)}=0$. Now, by using Eq. (123) we can show straightforwardly that there is no correction to the zero mode energy, at least at first-order approximation, i.e. $E_{0}^{(1)}=0$.

Let us consider now the case when $b_{3}=0$ and $a_{2}=\lambda \lesssim$ $10^{-2}$. Similarly, we find that the first-order corrections $U_{ \pm}^{(1)}$ are given in this case by,

$$
\begin{aligned}
U_{+}^{(1)}= & -\alpha^{2}\left(2-3 \operatorname{sech}^{2}(\alpha x)-2 \operatorname{sech}(\alpha x) \tanh (\alpha x)\right), \\
U_{-}^{(1)}= & -\alpha^{2} \beta^{2} e^{2 \operatorname{gd}(x)}\left(2-3 \operatorname{sech}^{2}(\alpha x)\right. \\
& -2 \operatorname{sech}(\alpha x) \tanh (\alpha x)) .
\end{aligned}
$$

Then, by substituting in Eq. (123) we find that the first-order correction to the zero mode also vanishes. Therefore, we can ensure that in the weak coupling regime, for $a_{2} \lesssim 10^{-2}$ and $b_{3} \lesssim 10^{-2}$, the stability of the extended ( $\mathrm{sG}+\mathrm{E}$ ) BPS solutions. Of course, for greater values of the coupling parameters, we should do a more complete analytical or numerical analysis of the spectral problem. We will leave this specific issue to be explored in other future work.

\section{Extended three-scalar fields models}

Now, we will construct some new three-scalar field extended models by applying a generalization of the extension method for three-field systems [2] to the one-field systems studied so far.

$6.1 \phi^{4}$ model coupled to the $\varphi^{6}$-like and the inverted $\zeta^{4 I}$ models

Let us start by considering the coupling of the standard $\phi^{4}$ model with the $\varphi^{6}$-like model, and also with the so-called the inverted $\zeta^{4 I}$-model [39]. The starting point is again the first-order equation

$\phi^{\prime}=W_{\phi}^{(1)}=\alpha\left(1-\phi^{2}\right)$,

together with the deformation functions,

$$
\begin{aligned}
\phi & =f_{1}(\varphi)=|\varphi|-1, \\
|\phi| & =f_{2}(\zeta)=\sqrt{1-\frac{\zeta^{2}}{\beta^{2}}}, \\
\zeta & =f_{3}(\varphi)=f_{1}^{-1}\left(f_{2}(\varphi)\right)=\beta \sqrt{1-(|\varphi|-1)^{2}},
\end{aligned}
$$

from which we have the corresponding the first-order equations for the deformed models,

$\varphi^{\prime}=\widetilde{W}_{\varphi}^{(1)}=\alpha \varphi(2-|\varphi|)$,
$\zeta^{\prime}=\widehat{W}_{\zeta}^{(1)}=-\alpha \omega \zeta \sqrt{1-\frac{\zeta^{2}}{\beta^{2}}}$

where we have defined $\omega=\operatorname{sgn}(\phi)$. Their corresponding static solutions are

$\phi(x)=\tanh (\alpha x), \quad \varphi_{ \pm}(x)= \pm(1+\tanh (\alpha x))$,

$\zeta(x)=\beta \operatorname{sech}(\alpha x)$.

Now, the main idea of the method can be straightforwardly generalized to three-fields. First, we write the right-hand side of Eq. (127) now in seven different and equivalent forms by using the deformation functions and their inverse functions, as follows

$$
\begin{aligned}
W_{\phi}^{(1)}(\phi) & =\alpha\left(1-\phi^{2}\right), \quad W_{\phi}^{(1)}(\zeta)=\frac{\alpha}{\beta^{2}} \zeta^{2}, \\
W_{\phi}^{(1)}(\varphi) & =\alpha\left[1-(|\varphi|-1)^{2}\right], \\
W_{\phi}^{(1)}(\phi, \varphi) & =\alpha[1-\phi(|\varphi|-1)], \\
W_{\phi}^{(1)}(\phi, \zeta) & =\frac{\alpha}{\beta} \zeta \sqrt{1-\phi^{2}}, \\
W_{\phi}^{(1)}(\varphi, \zeta) & =\frac{\alpha}{\beta} \zeta \sqrt{1-(|\varphi|-1)^{2}}, \\
W_{\phi}^{(1)}(\phi, \varphi, \zeta) & =\frac{\alpha}{\beta} \zeta \sqrt{1-\phi(|\varphi|-1)} .
\end{aligned}
$$

Similarly, for Eq. (131)

$$
\begin{aligned}
\widetilde{W}_{\varphi}^{(1)}(\zeta) & =\frac{\alpha}{\beta^{2}} \epsilon \zeta^{2}, \quad \widetilde{W}_{\varphi}^{(1)}(\phi)=\alpha \epsilon\left(1-\phi^{2}\right), \\
\widetilde{W}_{\varphi}^{(1)}(\varphi) & =\alpha \varphi(2-|\varphi|), \\
\widetilde{W}_{\varphi}^{(1)}(\phi, \varphi) & =\alpha \varphi(1-\phi), \\
\widetilde{W}_{\varphi}^{(1)}(\phi, \zeta) & =\frac{\alpha}{\beta} \epsilon \zeta \sqrt{1-\phi^{2}} \\
\widetilde{W}_{\varphi}^{(1)}(\varphi, \zeta) & =\frac{\alpha}{\beta} \epsilon \zeta \sqrt{1-(|\varphi|-1)^{2}} \\
\widetilde{W}_{\varphi}^{(1)}(\phi, \zeta, \varphi) & =\frac{\alpha}{\beta} \epsilon \zeta \sqrt{1-\phi(|\varphi|-1)}
\end{aligned}
$$

and for the Eq. (132) we have

$$
\begin{aligned}
\widehat{W}_{\zeta}^{(1)}(\zeta) & =-\alpha \omega \zeta \sqrt{1-\frac{\zeta^{2}}{\beta^{2}}} \\
\widehat{W}_{\zeta}^{(1)}(\phi) & =-\alpha \beta \phi \sqrt{1-\phi^{2}}, \quad \widehat{W}_{\zeta}^{(1)}(\phi, \zeta)=-\alpha \phi \zeta \\
\widehat{W}_{\zeta}^{(1)}(\varphi) & =-\alpha \beta(|\varphi|-1) \sqrt{1-(|\varphi|-1)^{2}} \\
\widehat{W}_{\zeta}^{(1)}(\phi, \varphi) & =-\alpha \beta \phi \sqrt{1-(|\varphi|-1)^{2}} \\
\widehat{W}_{\zeta}^{(1)}(\varphi, \zeta) & =-\alpha \zeta(|\varphi|-1) \\
\widehat{W}_{\zeta}^{(1)}(\phi, \zeta, \varphi) & =-\alpha \beta \omega \sqrt{1-\phi(|\varphi|-1)} \sqrt{1-\frac{\zeta^{2}}{\beta^{2}}}
\end{aligned}
$$

Now, we will use a generalization of the ansatz used in Eqs. (34) and (35) for the case of three-field systems, in the 
following form

$$
\begin{aligned}
& W_{\phi}^{(3)}(\phi, \varphi, \zeta) \\
& =a_{1} W_{\phi}^{(1)}(\varphi)+a_{2} W_{\phi}^{(1)}(\phi, \varphi)+a_{3} W_{\phi}^{(1)}(\phi) \\
& \quad+a_{4} W_{\phi}^{(1)}(\zeta)+a_{5} W_{\phi}^{(1)}(\phi, \zeta)+a_{6} W_{\phi}^{(1)}(\varphi, \zeta) \\
& \quad+a_{7} W_{\phi}^{(1)}(\phi, \varphi, \zeta)+p_{1} g(\varphi)+p_{2} g(\phi, \varphi)+p_{3} g(\phi) \\
& \quad+p_{4} g(\zeta)+p_{5} g(\phi, \zeta)+p_{6} g(\varphi, \zeta)+p_{7} g(\phi, \varphi, \zeta),
\end{aligned}
$$

$$
\begin{aligned}
& W_{\varphi}^{(3)}(\phi, \varphi, \zeta) \\
& =b_{1} \widetilde{W}_{\varphi}^{(1)}(\varphi)+b_{2} \widetilde{W}_{\varphi}^{(1)}(\phi, \varphi)+b_{3} \widetilde{W}_{\varphi}^{(1)}(\phi) \\
& \quad+b_{4} \widetilde{W}_{\varphi}^{(1)}(\zeta)+b_{5} \widetilde{W}_{\varphi}^{(1)}(\phi, \zeta)+b_{6} \widetilde{W}_{\varphi}^{(1)}(\varphi, \zeta) \\
& \quad+b_{7} \widetilde{W}_{\varphi}^{(1)}(\phi, \varphi, \zeta)+q_{1} \tilde{g}(\varphi)+q_{2} \tilde{g}(\phi, \varphi)+q_{3} \tilde{g}(\phi) \\
& \quad+q_{4} \tilde{g}(\zeta)+q_{5} \tilde{g}(\phi, \zeta)+q_{6} \tilde{g}(\varphi, \zeta)+q_{7} \tilde{g}(\phi, \varphi, \zeta),
\end{aligned}
$$

$$
\begin{aligned}
& W_{\zeta}^{(3)}(\phi, \varphi, \zeta) \\
&= c_{1} \widehat{W}_{\zeta}^{(1)}(\varphi)+c_{2} \widehat{W}_{\zeta}^{(1)}(\phi, \varphi)+c_{3} \widehat{W}_{\zeta}^{(1)}(\phi) \\
&+c_{4} \widehat{W}_{\zeta}^{(1)}(\zeta)+c_{5} \widehat{W}_{\zeta}^{(1)}(\phi, \zeta)+c_{6} \widehat{W}_{\zeta}^{(1)}(\varphi, \zeta) \\
&+c_{7} \widehat{W}_{\zeta}^{(1)}(\phi, \varphi, \zeta)+r_{1} \hat{g}(\varphi)+r_{2} \hat{g}(\phi, \varphi)+r_{3} \hat{g}(\phi) \\
&+r_{4} \hat{g}(\zeta)+r_{5} \hat{g}(\phi, \zeta)+r_{6} \hat{g}(\varphi, \zeta)+r_{7} \hat{g}(\phi, \varphi, \zeta),
\end{aligned}
$$

where the parameters must satisfy the following conditions

$$
\begin{aligned}
& \sum_{i=1}^{7} a_{i}=\sum_{i=1}^{7} b_{i}=\sum_{i=1}^{7} c_{i}=1, \\
& \sum_{i=1}^{7} p_{i}=\sum_{i=1}^{7} q_{i}=\sum_{i=1}^{7} r_{i}=0 .
\end{aligned}
$$

In addition, the $g$-functions are determined from solving the following constraints,

$W_{\phi \varphi}^{(3)}=W_{\varphi \phi}^{(3)}, \quad W_{\phi \zeta}^{(3)}=W_{\zeta \phi}^{(3)}, \quad W_{\varphi \zeta}^{(3)}=W_{\zeta \varphi}^{(3)}$.

After doing some long calculation, we get

$$
\begin{aligned}
W_{\phi}^{(3)}(\phi, \varphi, \zeta)= & \alpha\left(1-\phi^{2}\right)+a_{2} \phi(1+\phi-|\varphi|) \\
& -\frac{1}{2}\left(1-c_{6}\right)\left(\zeta^{2}-\beta^{2}\left(1-\phi^{2}\right)\right), \\
W_{\varphi}^{(3)}(\phi, \varphi, \zeta)= & \frac{\alpha \epsilon}{2}\left(\left(2+c_{6} \beta^{2}\right)\left(1-(|\varphi|-1)^{2}\right)\right. \\
& \left.-a_{2}\left(\phi^{2}-(|\varphi|-1)^{2}\right)-c_{6} \zeta^{2}\right), \\
W_{\zeta}^{(3)}(\phi, \varphi, \zeta)= & -\alpha \zeta\left(\phi-c_{6}(1+\phi-|\varphi|)\right) .
\end{aligned}
$$

After integrating, we finally obtain the following three-field superpotential

$$
\begin{aligned}
W^{(3)}(\phi, \varphi, \zeta)= & \alpha\left(1-a_{2}+\frac{\beta^{2}}{2}\left(1-c_{6}\right)\right) \phi\left(1-\frac{\phi^{2}}{3}\right) \\
& -\frac{\alpha}{2}\left(1-c_{6}\right) \phi \varphi^{2}-\frac{\alpha}{2} c_{6} \varphi^{2}(|\zeta|-1) \\
& +\alpha a_{2} \phi\left(1-\frac{\phi}{2}(|\zeta|-1)\right)+\frac{\alpha a_{2}}{2}|\zeta| \\
& +\frac{\alpha}{2}\left(2-a_{2}+\beta^{2} c_{6}\right) \zeta^{2}\left(1-\frac{|\zeta|}{3}\right) \\
& +\frac{\alpha}{12}\left(2 b_{4}+c_{6} \beta^{2}\right) .
\end{aligned}
$$

From now on we will named this three field model as the extended $\left(\phi^{4}+\varphi_{l}^{6}+\zeta^{4 I}\right)$ model, for which possesses the static configurations given in Eq. (133) are BPS solutions, with energy

$E_{\mathrm{BPS}}=\frac{2 \alpha}{3}\left(4+\beta^{2}\right)$.

The issue that arises from these results concerns the linear stability of the solutions for this superpotential. Although the stability analysis for three-field systems follows the same steps that the one presented in Sect. 5, it is actually further more complicated mostly because of the diagonalization of the Schrödinger-type operator, which is the key point in order to find the normal mode fluctuations. In this case, we will have

$$
\begin{aligned}
& \phi(x, t)=\phi_{s}(x)+\sum_{k} \rho_{k}(x) \cos \left(w_{k} t\right), \\
& \varphi(x, t)=\varphi_{s}(x)+\sum_{k} \sigma_{k}(x) \cos \left(w_{k} t\right), \\
& \zeta(x, t)=\zeta_{s}(x)+\sum_{k} \xi_{k}(x) \cos \left(w_{k} t\right),
\end{aligned}
$$

where $\rho_{k}, \sigma_{k}$, and $\xi_{k}$ are the fluctuations around the static solutions $\phi_{s}(x), \varphi_{s}(x)$, and $\zeta_{s}(x)$. Considering the dynamics of these three time-dependent fields up to first-order, we will obtain a corresponding Schrödinger-like equation $H \Psi_{k}(x)=$ $w_{k}^{2} \Psi_{k}(x)$,

$$
H=-\frac{d^{2}}{d x^{2}}+\left(\begin{array}{ccc}
V_{\phi \phi} & V_{\phi \varphi} & V_{\phi \zeta} \\
V_{\varphi \phi} & V_{\varphi \varphi} & V_{\varphi \zeta} \\
V_{\zeta \phi} & V_{\zeta \varphi} & V_{\zeta \zeta}
\end{array}\right)
$$

and

$\Psi_{k}(x)=\left(\begin{array}{c}\rho_{k}(x) \\ \sigma_{k}(x) \\ \xi_{k}(x)\end{array}\right)$.

For the case of BPS potentials, we can write this Hamiltonian in terms of linear operators, namely $H=A_{-}^{\dagger} A_{-}=A_{+} A_{-}$, where

$$
A_{ \pm}= \pm \frac{d}{d x}+\mathbf{W}, \quad \mathbf{W}=\left(\begin{array}{lll}
W_{\phi \phi} & W_{\phi \varphi} & W_{\phi \zeta} \\
W_{\varphi \phi} & W_{\varphi \varphi} & W_{\varphi \zeta} \\
W_{\zeta \phi} & W_{\zeta \varphi} & W_{\zeta \zeta}
\end{array}\right) .
$$


Our strategy again will be trying to diagonalize the matrix $\mathbf{W}$, and then the Schrödinger-type equation will be split into three equations, which will be analysed separately.

In the case of the BPS solutions (133) of the $\left(\phi^{4}+\varphi_{l}^{6}+\zeta^{4 I}\right)$ model (145), this matrix takes the following form

$\mathbf{W}=\tanh (\alpha x)\left(\begin{array}{ccc}-2 \alpha & 0 & 0 \\ 0 & -\alpha\left(2+\beta^{2}\right) & -\alpha \beta \epsilon \operatorname{csch}(\alpha x) \\ 0 & -\alpha \beta \epsilon \operatorname{csch}(\alpha x) & -\alpha\end{array}\right)$,

where we have chosen the parameters being $a_{2}=0$ e $c_{6}=1$ for simplicity. By computing its corresponding eigenvalues, we find

$$
\begin{aligned}
u_{0}= & -2 \alpha \tanh (\alpha x), \\
u_{ \pm}= & -\frac{\alpha}{2}\left(\left(3+\beta^{2}\right) \tanh (\alpha x)\right. \\
& \left. \pm \sqrt{4 \beta^{2}+\left(\beta^{2}-1\right)^{2} \tanh ^{2}(\alpha x)}\right) .
\end{aligned}
$$

Now, by setting $\beta=1$, we will find that the quantum mechanical potentials are given by,

$$
\begin{aligned}
U_{0} & =4 \alpha^{2}-6 \alpha^{2} \operatorname{sech}^{2}(\alpha x), \\
U_{ \pm} & =5 \alpha^{2}-6 \alpha^{2} \operatorname{sech}^{2}(\alpha x) \pm 4 \alpha^{2} \tanh (\alpha x),
\end{aligned}
$$

which are again Rosen-Morse II potentials (A.1). The $U_{0}$ potential has parameters $A=2 \alpha$ and $B=0$, and possesses eigenvalues $E_{0}=0$ and $E_{1}=3 \alpha^{2}$. The other two potentials $U_{ \pm}$have parameters $A=2 \alpha$ and $B= \pm 2 \alpha^{2}$ respectively, and only have the ground state $E_{0}=0$. Therefore, for these choice of parameters, we will have stability guaranteed. Another possible choice would be $a_{2}=0$ and $c_{6}=0$, however we will get essentially the same results.

\section{$6.2 \phi^{4}$ model coupled to sine-Gordon model and the \\ E-model}

Let us now construct a model obtained by the coupling of the standard $\phi^{4}$ model with sine-Gordon, and the E-model. The first-order equation for each one of these models are given by Eqs. (18), (25), and (27), namely

$$
\begin{aligned}
& \phi^{\prime}=W_{\phi}^{(1)}=\alpha\left(1-\phi^{2}\right), \\
& \chi^{\prime}=\widetilde{W}_{\chi}^{(1)}=\frac{\alpha}{\beta} \cos (\beta \chi), \\
& \eta^{\prime}=\widehat{W}_{\eta}^{(1)}=\alpha(1+\eta) \cos \left(\frac{1}{2} \ln (1+\eta)^{2}\right),
\end{aligned}
$$

with static solutions

$$
\begin{aligned}
& \phi(x)=\tanh (\alpha x), \quad \chi(x)=\frac{1}{\beta} \arctan (\sinh (\alpha x)), \\
& \eta(x)=\exp (\arctan (\sinh (\alpha x)))-1 .
\end{aligned}
$$

The deformation functions connecting the three models have the following forms,

$\phi=f_{1}(\chi)=\sin (\beta \chi)$,

$$
\begin{aligned}
\eta & =f_{2}(\chi)=e^{\beta \chi}-1, \\
\phi & =f_{3}(\eta)=f_{1}\left(f_{2}^{-1}(\eta)\right)=\sin \left(\frac{1}{2} \ln (1+\eta)^{2}\right) .
\end{aligned}
$$

By using these deformation functions and their inverse functions, we get the following expressions,

$$
\begin{aligned}
& W_{\phi}^{(1)}(\phi)=\alpha\left(1-\phi^{2}\right), \quad W_{\phi}^{(1)}(\chi)=\alpha \cos ^{2}(\beta \chi), \\
& W_{\phi}^{(1)}(\phi, \chi)=\alpha(1-\phi \sin (\beta \chi)), \\
& W_{\phi}^{(1)}(\eta, \chi)=\alpha\left[1-\sin (\beta \chi) \sin \left(\frac{1}{2} \ln (1+\eta)^{2}\right)\right], \\
& W_{\phi}^{(1)}(\eta)=\alpha \cos ^{2}\left(\frac{1}{2} \ln (1+\eta)^{2}\right), \\
& W_{\phi}^{(1)}(\phi, \eta)=\alpha\left[1-\phi \sin \left(\frac{1}{2} \ln (1+\eta)^{2}\right)\right], \\
& W_{\phi}^{(1)}(\phi, \eta, \chi)=\alpha\left[1-2 \phi \sin \left(\frac{\beta \chi}{2}\right) \cos \left(\frac{1}{4} \ln (1+\eta)^{2}\right)\right] .
\end{aligned}
$$

Similarly, we have

$$
\begin{aligned}
\widetilde{W}_{\chi}^{(1)}(\chi)= & \frac{\alpha}{\beta} \cos (\beta \chi), \quad \widetilde{W}_{\chi}^{(1)}(\phi)=\frac{\alpha}{\beta} \sqrt{1-\phi^{2}}, \\
\widetilde{W}_{\chi}^{(1)}(\eta)= & \frac{\alpha}{\beta} \cos \left(\frac{1}{2} \ln (1+\eta)^{2}\right), \\
\widetilde{W}_{\chi}^{(1)}(\phi, \chi)= & \frac{\alpha}{\beta}\left[\left(1-2 \phi^{2}\right) \cos (\beta \chi)\right. \\
& \left.+2 \phi \sqrt{1-\phi^{2}} \sin (\beta \chi)\right], \\
\widetilde{W}_{\chi}^{(1)}(\eta, \chi)= & \frac{\alpha}{\beta}\left[2 \cos \left(\frac{\beta \chi}{2}\right) \cos \left(\frac{1}{4} \ln (1+\eta)^{2}\right)-1\right], \\
\widetilde{W}_{\chi}^{(1)}(\phi, \eta)= & \frac{\alpha}{\beta}\left[\left(1-2 \phi^{2}\right) \cos \left(\frac{1}{2} \ln (1+\eta)^{2}\right)\right. \\
& \left.+2 \phi \sqrt{1-\phi^{2}} \sin \left(\frac{1}{2} \ln (1+\eta)^{2}\right)\right], \\
\widetilde{W}_{\chi}^{(1)}(\phi, \eta, \chi)= & \frac{\alpha}{\beta}\left[1-2 \phi \sin \left(\frac{\beta \chi}{2}\right) \cos \left(\frac{1}{4} \ln (1+\eta)^{2}\right)\right. \\
& \left.+2 \sqrt{1-\phi^{2}} \sin \left(\frac{\beta \chi}{2}\right) \sin \left(\frac{1}{4} \ln (1+\eta)^{2}\right)\right],
\end{aligned}
$$

and

$$
\begin{aligned}
\widehat{W}_{\eta}^{(1)}(\eta) & =\alpha(1+\eta) \cos \left(\frac{1}{2} \ln (1+\eta)^{2}\right), \\
\widehat{W}_{\eta}^{(1)}(\eta, \chi) & =\alpha(1+\eta) \cos (\beta \chi), \\
\widehat{W}_{\eta}^{(1)}(\chi) & =\alpha e^{\beta \chi} \cos (\beta \chi), \\
\widehat{W}_{\eta}^{(1)}(\phi, \chi)= & \alpha e^{\beta \chi} \sqrt{1-\phi^{2}}, \\
\widehat{W}_{\eta}^{(1)}(\phi)= & \alpha e^{\arcsin (\phi)} \sqrt{1-\phi^{2}}, \\
\widehat{W}_{\eta}^{(1)}(\phi, \eta)= & \alpha \sqrt{1-\phi^{2}}(1+\eta), \\
\widehat{W}_{\eta}^{(1)}(\phi, \eta, \chi)= & \alpha(1+\eta) \\
& \times\left[1-2 \phi \sin \left(\frac{\beta \chi}{2}\right) \cos \left(\frac{1}{4} \ln (1+\eta)^{2}\right)\right.
\end{aligned}
$$




$$
\left.+2 \sqrt{1-\phi^{2}} \sin \left(\frac{\beta \chi}{2}\right) \sin \left(\frac{1}{4} \ln (1+\eta)^{2}\right)\right]
$$

Now, from above parametrizations we can derive explicitly the functions $g, \tilde{g}$, and $\hat{g}$. After performing a long calculation, we find

$$
\begin{aligned}
W_{\phi}^{(3)}(\phi, \eta, \chi)= & \alpha\left(1-a_{4}-a_{5}\right)\left(1-\phi^{2}\right) \\
& +\alpha a_{4} \cos ^{2}\left(\frac{1}{2} \ln (1+\eta)^{2}\right) \\
& +\alpha a_{5}\left[1-\phi \sin \left(\frac{1}{2} \ln (1+\eta)^{2}\right)\right], \\
W_{\chi}^{(3)}(\phi, \eta, \chi)= & \frac{\alpha}{\beta} \cos (\beta \chi) \\
& -\frac{\alpha \beta c_{6}}{2} \sin (\beta \chi)\left((1+\eta)^{2}-e^{2 \beta \chi}\right) \\
& +\alpha \beta c_{1} e^{\beta \chi}\left(1+\eta-e^{\beta \chi}\right) \\
& \times(\cos (\beta \chi)-\sin (\beta \chi)), \\
W_{\eta}^{(3)}(\phi, \eta, \chi)= & \alpha\left(c_{1} e^{\beta \chi}+c_{6}(1+\eta)\right) \cos (\beta \chi) \\
& +\alpha\left(1-c_{1}-c_{6}\right)(1+\eta) \cos \left(\frac{1}{2} \ln (1+\eta)^{2}\right) \\
& +\frac{\alpha a_{4}}{(1+\eta)} \sin \left(\ln (1+\eta)^{2}\right) \\
& \times\left[\sin ^{2}\left(\frac{1}{2} \ln (1+\eta)^{2}\right)-\phi\right] \\
& +\frac{\alpha a_{5}}{2(1+\eta)} \cos \left(\frac{1}{2} \ln (1+\eta)^{2}\right) \\
& \times\left[\sin ^{2}\left(\frac{1}{2} \ln (1+\eta)^{2}\right)-\phi^{2}\right], \\
& {[16}
\end{aligned}
$$

which after being integrated lead us to the three-field superpotential

$$
\begin{aligned}
& W^{(3)}(\phi, \eta, \chi) \\
& =\alpha\left(1-a_{4}-a_{5}\right)\left(\phi-\frac{\phi^{3}}{3}\right)+\frac{\alpha}{\beta^{2}} \sin (\beta \chi) \\
& +\alpha a_{4}\left[\phi \cos ^{2}\left(\frac{1}{2} \ln (1+\eta)^{2}\right)+\frac{2}{3} \sin ^{3}\left(\frac{1}{2} \ln (1+\eta)^{2}\right)\right] \\
& +\frac{\alpha c_{1}}{5} e^{\beta \chi}\left[\left(5(1+\eta)-3 e^{\beta \chi}\right) \cos (\beta \chi)+e^{\beta \chi} \sin (\beta \chi)\right] \\
& +\frac{\alpha c_{6}}{10}\left[\left(5(1+\eta)^{2}-e^{2 \beta \chi}\right) \cos (\beta \chi)+2 e^{2 \beta \chi} \sin (\beta \chi)\right] \\
& +\frac{\alpha}{5}\left(1-c_{1}-c_{6}\right)(1+\eta)^{2}\left[2 \cos \left(\frac{1}{2} \ln (1+\eta)^{2}\right)\right. \\
& \left.+\sin ^{2}\left(\frac{1}{2} \ln (1+\eta)^{2}\right)\right]+\alpha a_{5}\left[\phi-\frac{\phi^{2}}{2} \sin \left(\frac{1}{2} \ln (1+\eta)^{2}\right)\right. \\
& \left.+\frac{1}{6} \sin ^{3}\left(\frac{1}{2} \ln (1+\eta)^{2}\right)\right] .
\end{aligned}
$$

This extended three-field superpotential describes the coupling of $\phi^{4}$, sine-Gordon, and the E-model, with the BPS solutions (157) connecting the minima $m_{1}=\left(-1,-\frac{\pi}{2 \beta},-1+\right.$ $\left.e^{-\pi / 2}\right)$ and $m_{2}=\left(+1,+\frac{\pi}{2 \beta},-1+e^{+\pi / 2}\right)$, with BPS energy

$E_{\mathrm{BPS}}=2 \alpha\left(\frac{2}{3}+\frac{1}{\beta^{2}}+\frac{1}{5} \cosh (\pi)\right)$

Now, regarding the linear stability of these BPS solutions, we compute the eigenvalues of the matrix $\mathbf{W}$ by choosing $a_{5}=-2 a_{4}$ and $c_{6}=-c_{1}$, leading us to

$$
\begin{aligned}
u_{0}= & -2 \alpha \tanh (\alpha x) \\
u_{ \pm}= & -\alpha \tanh (\alpha x) \\
& +\frac{\alpha}{2} \operatorname{sech}(\alpha x)\left[1-c_{1}\left(1+\beta^{2} e^{2 \operatorname{gd}(x)}\right)\right. \\
& \mp\left(1+2 c_{1}\left(\beta^{2} e^{2 \operatorname{gd}(x)}-1\right)\right. \\
& \left.\left.+c_{1}^{2}\left(1+\beta^{2} e^{-2 \operatorname{gd}(x)}\right)^{2}\right)^{1 / 2}\right]
\end{aligned}
$$

We see that the first quantum-mechanical potential derived from Eq. (169) is simply given by

$U_{0}(x)=4 \alpha^{2}-6 \alpha^{2} \operatorname{sech}^{2}(\alpha x)$,

whose energy eigenvalues are $E_{0}=0$ and $E_{1}=3 \alpha^{2}$, which partially guarantees stability. However, the others two potentials $U_{ \pm}$have complicated forms (see Fig. 11), which as a consequence arises some difficulties in obtaining analytical results, except for the $c_{1}=0$ case which decouples the sineGordon field. Instead of that, we will perform an approximated analysis for those cases. We see from the plots of the potentials in Fig. 12 that for small values of $c_{1}$ these potentials approximate to Rosen-Morse II and Scarf II profiles, respectively. Let us consider small values of the parameter, that is $c_{1}=\lambda \lesssim 10^{-2}$, so we obtain the approximated potentials up to first-order,

$U_{ \pm}(x)=U_{ \pm}^{(0)}(x)+\lambda U_{ \pm}^{(1)}(x)$,

where the unperturbed potentials are given by

$U_{+}^{(0)}(x)=\alpha^{2}-2 \alpha^{2} \operatorname{sech}^{2}(\alpha x)$,

$U_{-}^{(0)}(x)=\alpha^{2}-\alpha^{2} \operatorname{sech}^{2}(\alpha x)-3 \alpha^{2} \operatorname{sech}(\alpha x) \tanh (\alpha x)$,

while the first-order corrections are,

$U_{+}^{(1)}(x)=\alpha^{2} \beta^{2} e^{2 \operatorname{gd}(x)}(3 \operatorname{sech}(\alpha x) \tanh (\alpha x)$ $\left.-2 \operatorname{sech}^{2}(\alpha x)\right)$,

$U_{-}^{(1)}(x)=\alpha^{2}\left(3 \operatorname{sech}(\alpha x) \tanh (\alpha x)-2 \operatorname{sech}^{2}(\alpha x)\right)$.

We notice that the unperturbed potential $U_{+}^{(0)}$ is described by the potential Rosen-Morse II with $A=\alpha$ and $B=0$, while $U_{-}^{(0)}$ is described by the potential Scarf II with $A=\alpha$ and $B=-\alpha$. Both potentials only possess one bound state, the 

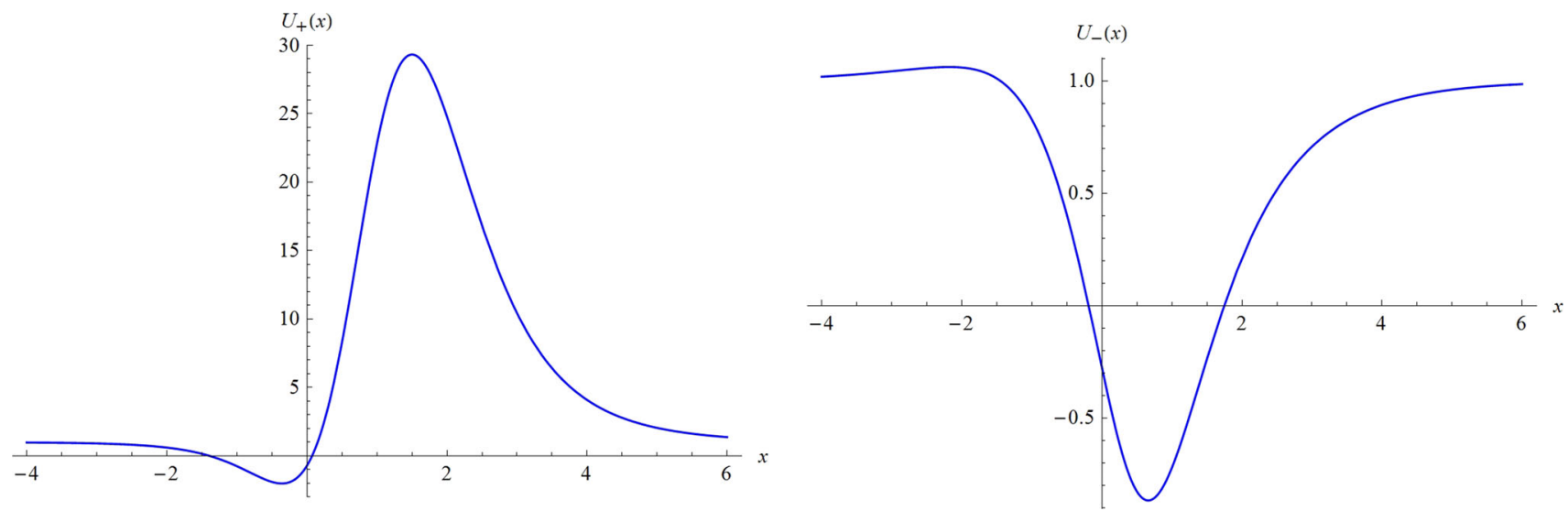

Fig. 11 Quantum-mechanical potentials $U_{+}$(on the left) and $U_{-}$(on the right) for $\alpha=\beta=c_{1}=1$
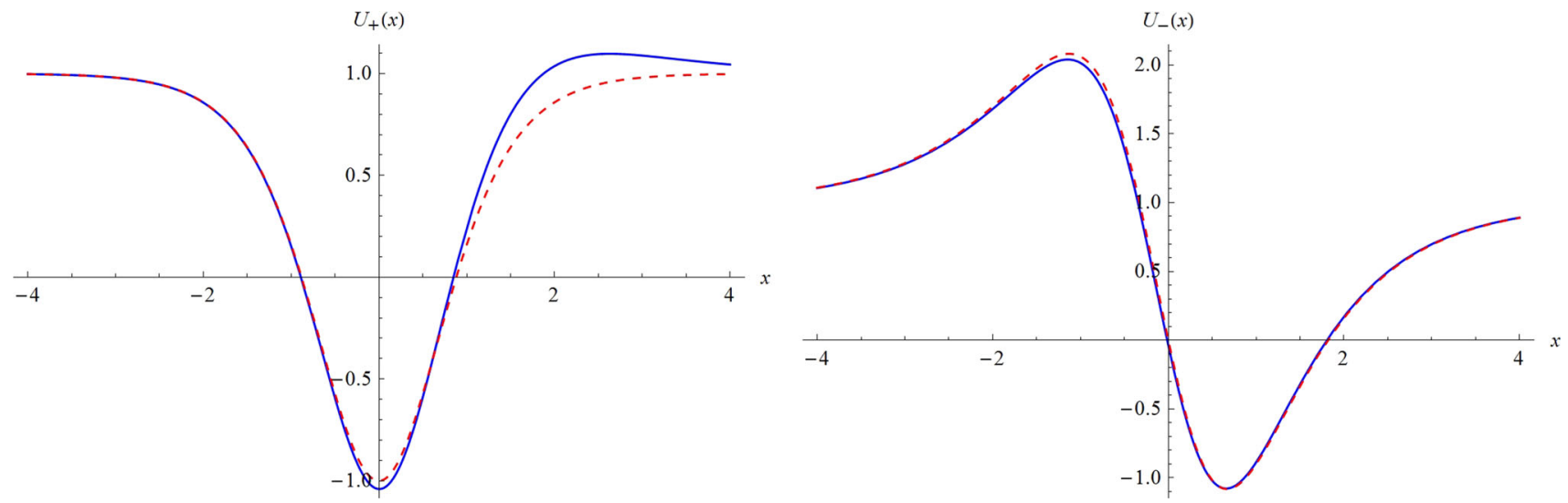

Fig. 12 Quantum-mechanical potentials $U_{+}$(on the left) and $U_{-}$(on the right) for $\alpha=\beta=1$. For both, we have plotted the values $c_{1}=0$ (dashed red lines) and $c_{1}=0.02$ (blue solid lines)

zero mode $E_{0}^{(0)}=0$. Therefore, the corresponding first-order correction $E_{0}^{(1)}$ to the zero energy will be obtained from

$E_{0}^{(1)}=\int_{-\infty}^{\infty} d a\left[\sigma_{0}^{*}(x) U_{+}^{(0)}(x) \sigma_{0}(x)+\xi_{0}^{*}(x) U_{-}^{(0)}(x) \xi_{0}(x)\right]$

where the zero mode eigenfunctions $\sigma_{0}(x)$ and $\xi_{0}(x)$ can be computed from Eqs. (A.4) and (A.8), respectively. Computing explicitly the integral we can easily verified that $E_{0}^{(1)}=0$, and then we can ensure the stability of the solutions up to first-order approximation.

It is clear that there is enough room for several different topological sectors depending on the values of the four arbitrary parameters. In particular, if we chose $c_{1}=c_{6}=0$, we get the following two BPS solutions,

$\eta^{( \pm)}(x)=-1+e^{ \pm \pi / 2}, \quad \chi(x)=\frac{1}{\beta} \arctan (\sinh (\alpha x))$,

$\phi^{( \pm)}(x)=\frac{\left(1-a_{4}\right) \pm e^{\left( \pm 2\left(1-a_{4}\right) \mp a_{5}\right) \alpha x}}{ \pm\left(a_{5}-\left(1-a_{4}\right)\right)+e^{\left( \pm 2\left(1-a_{4}\right) \mp a_{5}\right) \alpha x}}$, providing that $a_{5} \neq 2\left(1-a_{4}\right)$, and also that $a_{5}>\left(1-a_{4}\right)$ for $\phi^{(+)}(x)$, and $a_{5}<\left(1-a_{4}\right)$ for $\phi^{(-)}(x)$. The kink solutions for the $\phi^{4}$ field in Eq. (177) have the very same form as the ones previously found Eqs. (61) and (63) for the extended $\left(\phi^{4}+\mathrm{sG}\right)$ model, and also interpolates between the values \pm 1 and $\frac{ \pm\left(1-a_{4}\right)}{a_{5}-\left(1-a_{4}\right)}$, respectively. Depending on the values of the parameters, we will have two topological sectors with the corresponding BPS energies given by

$E_{\mathrm{BPS}}^{( \pm)}=\left|\frac{2 \alpha}{\beta^{2}} \pm \frac{\alpha}{6} \frac{\left(a_{5}-2\left(1-a_{4}\right)\right)^{3}}{\left(a_{5}-\left(1-a_{4}\right)\right)^{2}}\right|$.

By analysing the stability of the $\phi^{(-)}$solution, we will find that the associated $\mathbf{W}$ matrix reads,

$$
\mathbf{W}=\left(\begin{array}{ccc}
2 \alpha\left(a_{4}-1\right) \phi^{(-)} & 0 & 0 \\
0 & -\alpha \sin (\beta \chi) & 0 \\
0 & 0 & 2 \alpha e^{\pi} a_{4}\left(1+\phi^{(-)}\right)+\alpha
\end{array}\right)
$$

where we have chosen $a_{5}=0$ for simplicity, and therefore we have that $a_{4}<1$. The quantum-mechanical potentials 
will be given by

$$
\begin{aligned}
U_{0}(x)= & \alpha^{2}-2 \alpha^{2} \operatorname{sech}^{2}(\alpha x), \\
U_{-}(x)= & \frac{8 \alpha^{2} a_{4}\left(a_{4}-1\right)^{2} e^{\pi+2 \alpha\left(a_{4}-1\right) x}}{\left[1-a_{4}+e^{2 \alpha\left(a_{4}-1\right) x}\right]^{2}} \\
& +\left(\alpha-\frac{4 \alpha e^{\pi} a_{4}\left(a_{4}-1\right)}{1-a_{4}+e^{2 \alpha\left(a_{4}-1\right) x}}\right)^{2}, \\
U_{+}(x)= & 4 \alpha^{2}\left(a_{4}-1\right)^{2} \\
& \times \frac{\left(a_{4}-1\right)^{2}+4\left(a_{4}-1\right) e^{2 \alpha\left(a_{14}-1\right) x}+e^{4 \alpha\left(a_{4}-1\right) x}}{\left(1-a_{4}+e^{2 \alpha\left(a_{4}-1\right) x}\right)^{2}},
\end{aligned}
$$

from where we immediately see that the potential $U_{0}$ only possesses the eigenvalue $E_{0}=0$. In its turn, we can verify that the potentials $U_{ \pm}$can be described by shifted RosenMorse II potentials, namely

$$
\begin{aligned}
U_{ \pm}(x)= & A_{ \pm}^{2}+\frac{B_{ \pm}^{2}}{A_{ \pm}^{2}}-A_{ \pm}\left(A_{ \pm}+\kappa\right) \operatorname{sech}^{2}\left[\kappa\left(x-x_{0}^{ \pm}\right)\right] \\
& +2 B_{ \pm} \tanh \left[\kappa\left(x-x_{0}^{ \pm}\right)\right]
\end{aligned}
$$

where $\kappa=\alpha\left(1-a_{4}\right)$, and the parameters are

$A_{+}=2 \alpha\left(1-a_{4}\right), \quad B_{+}=0$,

$x_{0}^{+}=\frac{1}{2 \alpha\left(a_{4}-1\right)} \ln \left(1-a_{4}\right)$,

and

$A_{-}=-2 \alpha e^{\pi} a_{4}, \quad B_{-}=-2 \alpha^{2} e^{\pi} a_{4}\left(1+2 e^{\pi} a_{4}\right)$,

$x_{0}^{-}=\frac{1}{2 \alpha\left(a_{4}-1\right)} \ln \left(\frac{\left(1+4 e^{\pi}\right)^{2} a_{4}^{2}\left(1-a_{4}\right)}{\left(2-a_{4}\right)^{2}}\right)$.

We find that the potential $U_{+}$possesses the eigenvalues $E_{0}=$ 0 and $E_{1}=3 \alpha^{2}\left(1-a_{4}\right)^{2}$, whereas the potential $U_{-}$only possesses the eigenvalue $E_{0}=0$, if we have that

$-\frac{e^{-\pi}}{2}<a_{4}<-\frac{e^{-\pi}}{4}$.

Thus, this particular solution is stable only if the parameter $a_{4}$ satisfies the constraint (186), at least for our choice of parameters. Following an analogous procedure, the stability analysis of the solution $\phi^{(+)}$will lead us to similar results.

\section{$6.3 \phi^{4}$ model coupled to two sine-Gordon models}

In this last example, we will construct a three-field system that couples the $\phi^{4}$ field with two different sine-Gordon fields $\chi$ and $\psi$. The first-order equations are

$$
\begin{aligned}
\phi^{\prime}(x) & =W_{\phi}^{(1)}=\alpha\left(1-\phi^{2}\right), \\
\chi^{\prime}(x) & =\widetilde{W}_{\chi}^{(1)}=\frac{\alpha}{\beta} \cos (\beta \chi), \\
\psi^{\prime}(x) & =\widehat{W}_{\psi}^{(1)}=\frac{\alpha}{\gamma} \cos (\gamma \psi),
\end{aligned}
$$

and their corresponding static solutions are

$$
\begin{aligned}
& \phi(x)=\tanh (\alpha x), \quad \chi(x)=\frac{1}{\beta} \arctan (\sinh (\alpha x), \\
& \psi(x)=\frac{1}{\gamma} \arctan (\sinh (\alpha x) .
\end{aligned}
$$

The deformation functions are,

$\begin{aligned} \phi & =f_{1}(\chi)=\sin (\beta \chi), \\ \phi & =f_{2}(\psi)=\sin (\gamma \psi), \\ \chi & =f_{3}(\psi)=\frac{\gamma}{\beta} \psi .\end{aligned}$

As it was already done in the previous models, we use these functions to write the following equivalent expressions,

$$
\begin{aligned}
W_{\phi}^{(1)}(\phi) & =\alpha\left(1-\phi^{2}\right), \quad W_{\phi}^{(1)}(\chi)=\alpha \cos ^{2}(\beta \chi), \\
W_{\phi}^{(1)}(\psi) & =\alpha \cos ^{2}(\gamma \psi), \\
W_{\phi}^{(1)}(\phi, \chi) & =\alpha(1-\phi \sin (\beta \chi)) \\
W_{\phi}^{(1)}(\chi, \psi) & =\alpha(1-\sin (\beta \chi) \sin (\gamma \psi)) \\
W_{\phi}^{(1)}(\phi, \psi) & =\alpha(1-\phi \sin (\gamma \psi)) \\
W_{\phi}^{(1)}(\phi, \chi, \psi) & =\alpha\left(1-2 \phi \sin \left(\frac{\beta \chi}{2}\right) \cos \left(\frac{\gamma \psi}{2}\right)\right),
\end{aligned}
$$

and

$$
\begin{aligned}
\widetilde{W}_{\chi}^{(1)}(\chi) & =\frac{\alpha}{\beta} \cos (\beta \chi), \quad \widetilde{W}_{\chi}^{(1)}(\phi)=\frac{\alpha}{\beta} \sqrt{1-\phi^{2}}, \\
\widetilde{W}_{\chi}^{(1)}(\psi) & =\frac{\alpha}{\beta} \cos (\gamma \psi), \\
\widetilde{W}_{\chi}^{(1)}(\chi, \phi) & =\frac{\alpha}{\beta} \sqrt{1-\phi \sin (\beta \chi)}, \\
\widetilde{W}_{\chi}^{(1)}(\phi, \psi) & =\frac{\alpha}{\beta} \sqrt{1-\phi \sin (\gamma \psi)}, \\
\widetilde{W}_{\chi}^{(1)}(\chi, \psi) & =\frac{\alpha}{\beta} \sqrt{1-\sin (\beta \chi) \sin (\gamma \psi)} \\
\widetilde{W}_{\chi}^{(1)}(\phi, \chi, \psi) & =\frac{\alpha}{\beta}\left(\cos (\beta \chi) \cos ^{2}(\gamma \psi)-\phi^{2} \cos (\beta \chi)\right. \\
& +2 \phi \sin (\beta \chi) \cos (\gamma \psi)),
\end{aligned}
$$

and also

$$
\begin{aligned}
\widehat{W}_{\psi}^{(1)}(\psi) & =\frac{\alpha}{\gamma} \cos (\gamma \psi), \quad \widehat{W}_{\psi}^{(1)}(\phi)=\frac{\alpha}{\gamma} \sqrt{1-\phi^{2}}, \\
\widehat{W}_{\psi}^{(1)}(\chi) & =\frac{\alpha}{\gamma} \cos (\beta \chi), \\
\widehat{W}_{\psi}^{(1)}(\psi, \phi) & =\frac{\alpha}{\gamma} \sqrt{1-\phi \sin (\gamma \psi),} \\
\widehat{W}_{\psi}^{(1)}(\phi, \chi) & =\frac{\alpha}{\gamma} \sqrt{1-\phi \sin (\beta \chi)}, \\
\widehat{W}_{\psi}^{(1)}(\chi, \psi) & =\frac{\alpha}{\gamma} \sqrt{1-\sin (\beta \chi) \sin (\gamma \psi),}
\end{aligned}
$$


$\widehat{W}_{\psi}^{(1)}(\phi, \chi, \psi)=\frac{\alpha}{\gamma}\left(\cos (\gamma \psi) \cos ^{2}(\beta \chi)-\phi^{2} \cos (\gamma \psi)\right.$

$+2 \phi \sin (\gamma \psi) \cos (\beta \chi))$.

(196)

As before, we use all of these expressions to obtain the corresponding $g$-functions, and then by substituting the results in Eqs. (137)-(139), we have

$$
\begin{aligned}
W_{\phi}^{(3)}(\phi, \chi, \psi)= & \alpha\left(1-\phi^{2}\right)+\alpha a_{1}\left(\phi^{2}-\sin ^{2}(\beta \chi)\right) \\
& +\alpha a_{4}\left(\phi^{2}-\sin ^{2}(\gamma \psi)\right) \\
& +\alpha a_{2} \phi(\phi-\sin (\beta \chi)) \\
& +\alpha a_{5} \phi(\phi-\sin (\gamma \psi)), \\
W_{\chi}^{(3)}(\phi, \chi, \psi)= & \frac{\alpha}{\beta}\left(1-b_{4}\right) \cos (\beta \chi)+\frac{\alpha b_{4}}{\beta} \cos (\gamma \psi) \\
& -\alpha \beta a_{1} \phi \sin (2 \beta \chi)-\frac{\alpha \beta a_{2}}{2} \phi^{2} \cos (\beta \chi) \\
& +\alpha \beta\left(2 a_{1}+\frac{a_{2}}{2}\right) \sin ^{2}(\beta \chi) \cos (\beta \chi), \\
W_{\psi}^{(3)}(\phi, \chi, \psi)= & \frac{\alpha}{\gamma} \cos (\gamma \psi)+\frac{\alpha \gamma b_{4}}{\beta^{2}}(\gamma \psi-\beta \chi) \sin (\gamma \psi) \\
& -\alpha \gamma a_{4} \phi \sin (2 \gamma \psi)-\frac{\alpha \gamma a_{5}}{2} \phi^{2} \cos (\gamma \psi) \\
& +\alpha \gamma\left(2 a_{4}+\frac{a_{5}}{2}\right) \sin ^{2}(\gamma \psi) \cos (\gamma \psi),
\end{aligned}
$$

with the corresponding superpotential given by

$$
\begin{aligned}
W^{(3)}(\phi, \chi, \psi)= & \alpha \phi-\alpha\left(1-a_{1}-a_{2}-a_{4}-a_{5}\right) \frac{\phi^{3}}{3} \\
& -\alpha a_{1} \phi \sin ^{2}(\beta \chi)-\frac{\alpha a_{2}}{2} \phi^{2} \sin (\beta \chi) \\
& -\alpha a_{4} \phi \sin ^{2}(\gamma \psi)-\frac{\alpha a_{5}}{2} \phi^{2} \sin (\gamma \psi) \\
& +\frac{\alpha}{\beta^{2}}\left(1-b_{4}\right) \sin (\beta \chi) \\
& +\frac{\alpha}{3}\left(2 a_{1}+\frac{a_{2}}{2}\right) \sin ^{3}(\beta \chi) \\
& +\frac{\alpha b_{4}}{\beta^{2}}(\beta \chi-\gamma \psi) \cos (\gamma \psi) \\
& +\frac{\alpha}{3}\left(2 a_{4}+\frac{a_{5}}{2}\right) \sin ^{3}(\gamma \psi) \\
& +\frac{\alpha}{\gamma^{2}}\left(1+\frac{b_{4} \gamma^{2}}{\beta^{2}}\right) \sin (\gamma \psi) .
\end{aligned}
$$

This new extended three-field superpotential describes the coupling of the $\phi^{4}$ field with two different sine-Gordon fields, and will be named as the extended $\left(\phi^{4}+\mathrm{sG}_{1}+\mathrm{sG}_{2}\right)$ model. The static solutions (190) are BPS solutions of its first-order equations, connecting the minima $m_{1}=\left(-1,-\frac{\pi}{2 \beta},-\frac{\pi}{2 \gamma}\right)$ and $m_{2}=\left(1, \frac{\pi}{2 \beta}, \frac{\pi}{2 \gamma}\right)$, with BPS energy given by

$E_{\mathrm{BPS}}=2 \alpha\left(\frac{2}{3}+\frac{1}{\beta^{2}}+\frac{1}{\gamma^{2}}\right)$.
Now, in order to analyse linear stability of the BPS solutions (190), we find that in this case the corresponding matrix W takes the following form,

$\mathbf{W}=\tanh (\alpha x)\left(\begin{array}{ccc}-2 \alpha & 0 & 0 \\ 0 & \alpha\left(b_{4}-1\right) & -\frac{\alpha \gamma b_{4}}{\beta} \\ 0 & -\frac{\alpha \gamma b_{4}}{\beta} & \alpha\left(\frac{\gamma^{2} b_{4}}{\beta^{2}}-1\right)\end{array}\right)$,

where we have chosen $a_{5}=-2 a_{4}$ and $a_{2}=-2 a_{1}$, for simplicity. By diagonalizing this matrix, we will find the following eigenvalues

$u_{0}=-2 \alpha \tanh (\alpha x), \quad u_{+}=-\alpha \tanh (\alpha x)$,

$u_{-}=-\mu \tanh (\alpha x)$,

where in this case the parameter $\mu=\alpha-\alpha b_{4}\left(1+\frac{\gamma^{2}}{\beta^{2}}\right)$. Then, the corresponding quantum-mechanical potentials will be given as follows,

$U_{0}=4 \alpha^{2}-6 \alpha^{2} \operatorname{sech}^{2}(\alpha x)$,

$U_{+}=\alpha^{2}-2 \alpha^{2} \operatorname{sech}^{2}(\alpha x)$,

$U_{-}=\mu^{2}-\mu(\mu+\alpha) \operatorname{sech}^{2}(\alpha x)$.

which are again Rosen-Morse II potentials. We see that the potential $U_{0}$ is the same as the one in Eq. (153), and has the eigenvalues $E_{0}=0$ and $E_{1}=3 \alpha^{2}$. The parameters for the potential $U_{+}$are $A=\alpha$ and $B=0$, and has only one eigenvalue, $E_{0}=0$. For the potential $U_{-}$the parameters are $A=\mu$ and $B=0$. In this case, the number of eigenvalues will be now constrained by $0 \leq k<1-b_{4}\left(1+\frac{\gamma^{2}}{\beta^{2}}\right)$, which requires that $b_{4}<\frac{\beta^{2}}{\left(\beta^{2}+\gamma^{2}\right)}$ in order to guarantee stability. Therefore, when $0<b_{4}<\frac{\beta^{2}}{\left(\beta^{2}+\gamma^{2}\right)}$ there exists only one eigenvalue $E_{0}=0$. For $b_{4}<0$, we note that the number of bound states increases for decreasing $b_{4}$, and then the potential could have more than one non-negative eigenvalue, guaranteeing in this way the stability of the BPS solutions.

There are also several others interesting features that can be mentioned about this new model. For instance, the projection of the corresponding potential in the $(\chi, \psi)$ plane gives the following,

$$
\begin{aligned}
V(0, \chi, \psi) \\
=\frac{\alpha^{2}}{2}\left[\frac{1}{\beta^{2}}\left(1-b_{4}+2 a_{1} \beta^{2}\right)^{2} \cos ^{2}(\beta \chi)\right. \\
+\frac{1}{\beta^{2} \gamma^{2}}\left(\frac{b_{4}^{2}}{\beta^{2}}+\frac{\left(1+2 \gamma^{2}\left(1-a_{1}\right)\right)^{2}}{\gamma^{2}}\right) \cos ^{2}(\gamma \psi) \\
+\frac{2 b_{4}}{\beta^{2}}\left(1-b_{4}+2 a_{1} \beta^{2}\right) \cos (\beta \chi) \cos (\gamma \psi) \\
+2 a_{1}\left(1-a_{1}\right) \cos ^{2}(\beta \chi) \cos ^{2}(\gamma \psi) \\
-4 a_{1} b_{4} \cos ^{3}(\beta \chi) \cos (\gamma \psi) \\
+a_{1}\left(a_{1}+4\left(b_{4}-1\right)-8 a_{1} \beta^{2}\right) \cos ^{4}(\beta \chi)
\end{aligned}
$$




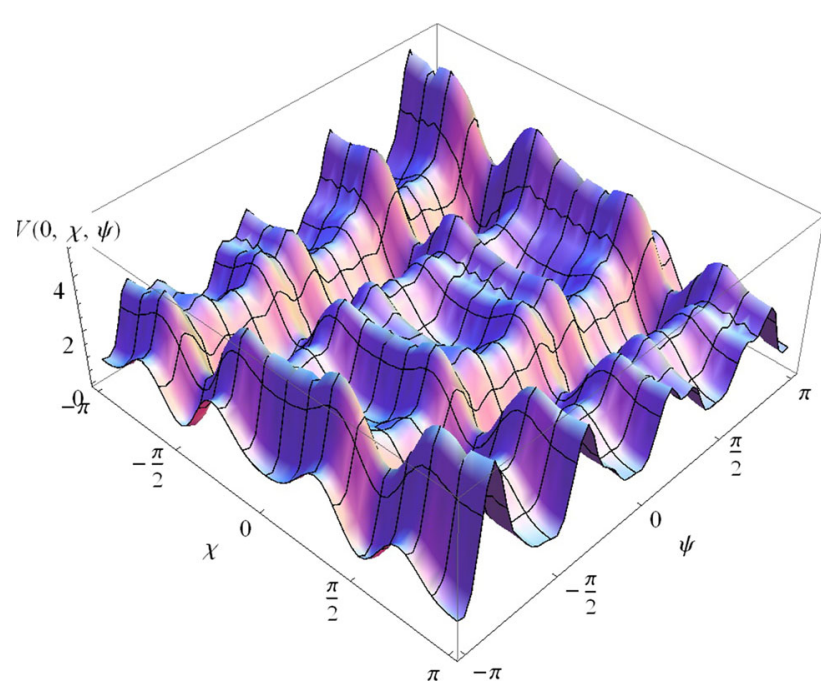

Fig. 13 Plot of the projection of the potential $V(0, \chi, \psi)$ for the values $\alpha=1, \beta=1, \gamma=2, a_{1}=2$ and $b_{4}=0.1$

$$
\begin{aligned}
& +\left(1-a_{1}\right)\left(\left(1-a_{1}\right)\left(1-8 \gamma^{2}\right)-4\right) \cos ^{4}(\gamma \psi) \\
& +4 a_{1}^{2} \beta^{2} \cos ^{6}(\beta \chi)+4 \gamma^{2}\left(1-a_{1}\right)^{2} \cos ^{6}(\gamma \psi) \\
& -\frac{b_{4}}{\beta^{2}}(\beta \chi-\gamma \psi) \sin (2 \gamma \psi)\left(1+2\left(1-a_{1}\right) \gamma^{2} \sin ^{2}(\gamma \psi)\right) \\
& \left.+\frac{\gamma^{2} b_{4}^{2}}{\beta^{4}}(\beta \chi-\gamma \psi)^{2} \sin ^{2}(\gamma \psi)\right],
\end{aligned}
$$

where we have considered $a_{2}=a_{5}=0$, and $a_{4}=1-a_{1}$, without loss of generality. It is worth pointing out that this potential is not BPS, and even though its minima are located at

$m_{k}=\left(\frac{\pi}{2 \beta}(2 k-1), \frac{\pi}{2 \gamma}(2 k-1)\right), \quad k \in \mathbb{Z}$,

the static sine-Gordon kinks are no longer solutions of its field equations. Despite of being an interesting potential (see Fig. 13), we have not been able to find any explicit analytical solutions for it. It would be interesting to look for at least numerical solutions and also further explore this potential. That could be addressed in more detail in another work.

On the other hand, when substituting $\phi= \pm 1$ directly in (200), and setting $a_{1}=a_{2}=a_{4}=a_{5}=0$, we end up with a different effective two-fields superpotential, and its corresponding potential (Fig. 14), given by

$$
\begin{aligned}
W_{\mathrm{eff}}^{(2)}(\chi, \psi)= & \frac{\alpha\left(1-b_{4}\right)}{\beta^{2}} \sin (\beta \chi)+\alpha\left(\frac{1}{\gamma^{2}}+\frac{b_{4}}{\beta^{2}}\right) \sin (\gamma \psi) \\
& +\frac{\alpha b_{4}}{\beta^{2}}(\beta \chi-\gamma \psi) \cos (\gamma \psi) \\
V_{\mathrm{eff}}(\chi, \psi)= & \frac{\alpha^{2}}{2}\left[\frac{1}{\beta^{2}}\left(1-b_{4}\right)^{2} \cos ^{2}(\beta \chi)\right. \\
& +\left(\frac{b_{4}^{2}}{\beta^{2}}+\frac{1}{\gamma^{2}}\right) \cos ^{2}(\gamma \psi)
\end{aligned}
$$

$$
\begin{aligned}
& -\frac{b_{4}}{\beta^{2}}(\beta \chi-\gamma \psi) \sin (2 \gamma \psi) \\
& +\frac{2 b_{4}}{\beta^{2}}\left(1-b_{4}\right) \cos (\beta \chi) \cos (\gamma \psi) \\
& \left.+\frac{\gamma^{2} b_{4}^{2}}{\beta^{4}}(\beta \chi-\gamma \psi)^{2} \sin ^{2}(\gamma \psi)\right] .
\end{aligned}
$$

Note that although this potential is somehow contained within the projection $V(0, \chi, \psi)$, they are actually different even if we set $a_{1}=0$ in Eq. (205), and in this case the static solutions for the sine-Gordon fields given in (190) are BPS solutions of the first-order equation for the effective superpotential (207). It is worth also noting that the simple coupling between the two sine-Gordon fields contained in the last term of Eq. (207) differs from some models previously constructed in the literature. In particular, if we eliminate the coupling term by setting $b_{4}=0$, then our potential will take the form of the non-integrable two-frequency sine-Gordon model considered in [59], where the authors studied how the particle spectrum of the model changes by considering the second interaction as a perturbation of the original integrable sine-Gordon model. In addition, after proper redefinitions

our superpotential (207), also with $b_{4}=0$, can be also seen as a limit case of the FKZ (Ferreira, Klimas, and Zakrewski) pre-potential based on the $S U$ (3) Lie algebra ${ }^{2}$ [3]. However, their potential $V$ will be quite different since a constant, real and positive-definite matrix $\eta_{a b}$, which is basically a modified version of the associated Cartan matrix, is directly involved in the definition of the FKZ models. Despite of these differences, it would be interesting to analyse if there exist any common points between the two methods of constructing multi-scalar field theories. This issue will be addressed in future investigations.

\section{Final remarks}

In this paper, we have presented the explicit construction of several interesting new models described by two and three real scalar fields theories in $(1+1)$-dimensions supporting BPS states. The way of constructing such field theories is called the extension method, which was introduced originally in $[1,2]$. This method requires considering initially several (not necessarily different) one-field systems which are known to support BPS states, and that are also connected through some mappings called deformation functions. Then, the corresponding first-order equations are rewritten in several different but equivalent non-trivial ways by using such functions and their inverse functions. Doing that, the fields are then coupled by introducing an ansatz for the first-order equa-

\footnotetext{
2 In fact, the model contains three parameters $\gamma_{1}, \gamma_{2}$, and $\gamma_{3}$, and then the exact equivalence will requires that the latter one vanishes.
} 

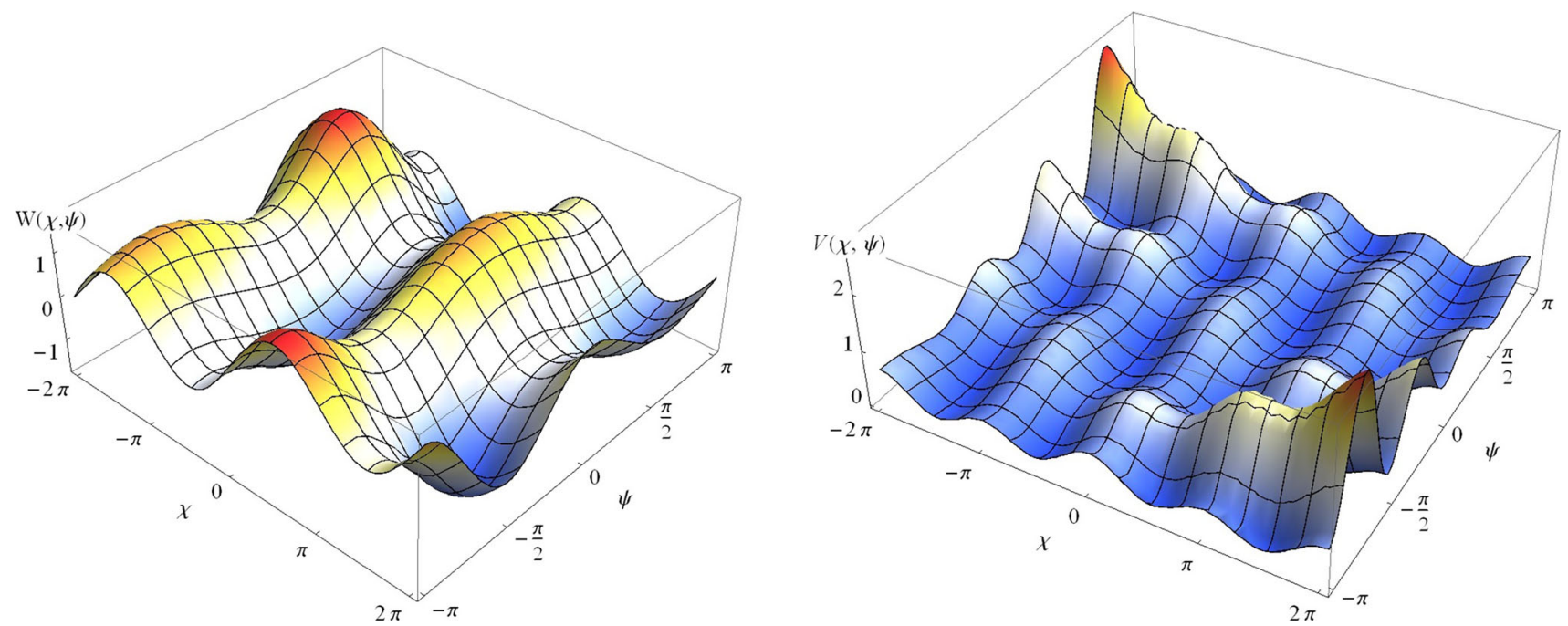

Fig. 14 Effective two-fields superpotential (on the left) and the associated potential (on the right) for the two coupled sine-Gordon fields. For both, we have plotted the values $\alpha=1, \beta=1, \gamma=2$, and $b_{4}=0.1$

tions for the resulting two-field model Eqs. (34) and (35), and respectively for three-field model Eqs. (137)-(139). To finish the procedure, some functions, called here as $g$-functions, are then introduced in order to guarantee smoothness of the superpotential, which are properly derived from consistency constraints (141).

The constructed theories were obtained by coupling basically some known one-scalar field BPS models, namely $\phi^{4}$ model, the $\varphi^{6}$-like model, the sine-Gordon model, the Emodel, and finally the inverse $\zeta^{4 I}$ model. One of the most important advantages of this method of constructing multifields models is that it maintains the BPS solutions of the original one-field systems. However, they are not the only possible BPS solutions for the multi-field superpotential. In fact, in some cases we have been able to find analytically (or numerically) other BPS solutions for the resulting model. We have also studied in some details the linear stability of the BPS states for the resulting multi-scalar superpotential. In general, these studies lead us with two very-well known exactly solvable quantum-mechanical problems, the RosenMorse II and the Scarf II potentials. For several choices of the potential parameters we have been able to perform analytically such analysis, and have found that they are stable with respect of small perturbations. However, in some cases the problem is somehow complicated and we have only been able to study in a qualitative and approximated way, with no full guarantee of the stability. Of course, such analysis could be improved by performing proper numerical simulations. Those investigations represent the next step in our studies on multi-scalar field theories and will be done in future works.

There are several other interesting issues that can be addressed in next investigations from our results. For instance, a more complete numerical study of the solutions and their stability, specially two-solitons solutions, would provide a good scenario for investigating the behaviour during kink collisions. In addition, that kind of analysis also could bring some additional information that allow us identify possible quasi-integrable multi-scalar models [60,61]. In particular, we are interested in the two coupled sine-Gordon model obtained in Sect. 6.3, which are slightly related to the FKZ models. We believe that a more detailed analysis would give some interesting connections between the two methods and probably help us answer some unsolved problems from both sides.

Finally, one more question of interest involves the investigations of possible supersymmetric generalization of the extension method. As it is well-known the interest on the study of supersymmetric kinks has a long history, and essentially concerns with the calculations of quantum corrections to the kink mass, and the central charge [44,62-66]. Therefore, it will be interesting to construct general supersymmetric field theories by using the extension method, especially for the ones that possess intrinsically infinite number of degenerate vacua, as it is the case of sine-Gordon and the E-model. These issues are also currently under investigations.

Acknowledgements Authors would like to thank to CAPES-Brazil for financial support. Authors are also grateful to the Directorate of Innovation and Research of the Federal University of Itajubá (DIPUNIFEI) for partial financial support at the very initial stage of this project.

Data Availability Statement This manuscript has no associated data or the data will not be deposited. [Authors' comment: This is a theoretical study and no experimental data has been listed.]

Open Access This article is licensed under a Creative Commons Attribution 4.0 International License, which permits use, sharing, adaptation, distribution and reproduction in any medium or format, as long as you 
give appropriate credit to the original author(s) and the source, provide a link to the Creative Commons licence, and indicate if changes were made. The images or other third party material in this article are included in the article's Creative Commons licence, unless indicated otherwise in a credit line to the material. If material is not included in the article's Creative Commons licence and your intended use is not permitted by statutory regulation or exceeds the permitted use, you will need to obtain permission directly from the copyright holder. To view a copy of this licence, visit http://creativecomm ons.org/licenses/by/4.0/.

Funded by SCOAP ${ }^{3}$.

\section{Appendix A: Associated exactly solvable potentials}

The very well-known exactly solvable Rosen-Morse II potential (or modified Pö̈chl-Teller potential) can be written in the following form [54],

$$
\begin{aligned}
U(x)= & A^{2}+\frac{B^{2}}{A^{2}}-A(A+\alpha) \operatorname{sech}^{2}(\alpha x) \\
& +2 B \tanh (\alpha x),
\end{aligned}
$$

where $\alpha>0$, and $A$ and $B$ are arbitrary real parameters. The bound states have the following eigenvalues,

$$
\begin{gathered}
E_{k}=A^{2}+\frac{B^{2}}{A^{2}}-(A-k \alpha)^{2}-\frac{B^{2}}{(A-k \alpha)^{2}}, \\
0 \leq k<\frac{(A-\sqrt{|B|})}{\alpha} .
\end{gathered}
$$

By imposing the stability condition, we find that

$A>0$, and $|B|<A^{2}$.

In addition, the corresponding wave eigenfunctions are given by

$$
\begin{aligned}
\psi_{k}(x)= & (1-\tanh (\alpha x))^{\frac{(s+t-k)}{2}}(1+\tanh (\alpha x))^{\frac{(s-t-k)}{2}} \\
& \times P_{k}^{(s+t-k, s-t-k)}(\tanh (\alpha x)),
\end{aligned}
$$

where $P_{k}^{(\alpha, \beta)}$ are the Jacobi polynomials, and

$s=A / \alpha, \quad t=\frac{B}{\alpha^{2}(s-k)}$.

Now, let us consider another very well-known exactly solvable potential, namely the Scarf II potential [54],

$$
\begin{aligned}
U(x)= & A^{2}+\left(B^{2}-A(A+\alpha)\right) \operatorname{sech}^{2}(\alpha x) \\
& +B(2 A+\alpha) \operatorname{sech}(\alpha x) \tanh (\alpha x),
\end{aligned}
$$

where $\alpha, A$, and $B$ are real parameters. Its corresponding bound states possess energy eigenvalues given by

$E_{k}=A^{2}-(A-k \alpha)^{2}, \quad 0 \leq k<\frac{A}{\alpha}$.

Their associated eigenfunctions can be written as follows,

$$
\begin{aligned}
\psi_{k}(x)= & i^{k}(\operatorname{sech}(\alpha x))^{s} e^{-u \arctan (\sinh (\alpha x))} \\
& \times P_{k}^{(i u-s-1 / 2,-i u-s-1 / 2)}(i \sinh (\alpha x)),
\end{aligned}
$$

where $P_{k}^{(\alpha, \beta)}$ are again the Jacobi polynomials, $s=A / \alpha$, and $u=B / \alpha$. It is clear that both potentials (A.1) and (A.6) coincides when $B=0$.

\section{References}

1. D. Bazeia, L. Losano, J.R.L. Santos, Kinklike structures in scalar field theories: from one-field to two-field models. Phys. Lett. A 377, 1615 (2013). arXiv:1304.6904 [hep-th]

2. J.R.L. Santos, P.H.R.S. Moraes, D.A. Ferreira, D.C.Vilar Neta, Building analytical three-field cosmological models. Eur. Phys. J. C 78, 169 (2018). arXiv:1707.02611 [hep-th]

3. L.A. Ferreia, P. Klimas, W.J. Zakrzewski, Self-dual sectors for scalar field theories in $(1+1)$ dimensions. JHEP 01, 020 (2019). arXiv: 1808.10052 [hep-th]

4. V.A. Gani, M.A. Lizunova, R.V. Radomskiy, Scalar triplet on a domain wall: an exact solution. JHEP 04, 043 (2016). arXiv: 1601.07954 [hep-th]

5. A. Alonso-Izquierdo, D. Bazeia, L. Losano, J. Mateos Guilarte, New models for two real scalar fields and their kink-like solutions. Adv. High Energy Phys. 2013, 183295 (2013). arXiv:1308.2724 [hep-th]

6. A. Alonso-Izquierdo, Kink dynamics in a system of two coupled scalar fields in two space-time dimensions. Physica D 365, 12-26 (2018). arXiv:1711.08784 [hep-th]

7. A. Alonso-Izquierdo, Non-topological kink scattering in a twocomponent scalar field theory model. Commun. Nonlinear Sci. Numer. Simul. 85, 105251 (2020). arXiv:1906.05040 [hep-th]

8. P.H.R.S. Moraes, J.R.L. Santos, Two scalar field cosmology from coupled one-field models. Phys. Rev. D 89(8), 083516 (2014). arXiv: 1403.5009 [gr-qc]

9. J.R.L. Santos, A. Souza De Dutra, O.C. Winter, R.A.C. Correa, A general method for transforming nonphysical configurations in BPS states. Adv. High Energy Phys. 2019, 5431067 (2019). arXiv:1809.04661 [hep-th]

10. A. Paliathanasis, G. Leon, S. Pan, Exact solutions in chiral cosmology. Gen. Relativ. Gravit. 51(9), 106 (2019). arXiv:1811.10038 [gr-qc]

11. N. Dimakis, A. Paliathanasis, P.A. Terzis, T. Christodoulakis, Cosmological solutions in multiscalar field theory. Eur. Phys. J. C 79(7), 618 (2019). arXiv:1904.09713 [gr-qc]

12. F.A. Brito, L. Losano, J.R.L. Santos, The extension method for Bloch branes. arXiv:1911.00191 [hep-th]

13. C. Adam, L.A. Ferreira, E. da Hora, A. Wereszczynski, W.J. Zakrzewski, Some aspects of self-duality and generalised BPS theories. JHEP 08, 062 (2013). arXiv:1305.7239

14. G. Luchini, T. Tassis, BPS states for scalar field theories based on $\mathfrak{g}_{2}$ and $\mathfrak{s u}(4)$ algebras. JHEP 05, 011 (2020). arXiv:1909.04467 [hep-th]

15. P. Klimas, W.J. Zakrzewski, Further comments on BPS systems. arXiv: 1908.02100 [hep-th]

16. P.G. Kevrekidis, J. Cuevas-Maraver (eds.), A Dynamical Perspective on the $\phi^{4}$ Model: Past, Present and Future. Part of the Nonlinear Systems and Complexity Book Series, vol. 26 (Springer, Cham, 2019)

17. J. Cuevas-Maraver, P.G. Kevrekidis, F. Williams (eds.), The SineGordon Model and Its Applications. Part of the Nonlinear Systems and Complexity Book Series, vol. 10 (Springer, Cham, 2014)

18. D.K. Campbell, J.F. Schonfeld, C.A. Wingate, Resonance structure in kink-antikink interactions in $\phi^{4}$ theory. Physica D 9, 1 (1983)

19. R.H. Goodman, R. Haberman, Kink-antikink collisions in the $\phi^{4}$ equation: the n-bounce resonance and the separatrix map. SIAM J. Appl. Dyn. Syst. 4, 1195 (2005) 
20. D.K. Campbell, M. Peyrard, P. Sodano, Kink-antikink interactions in the double sine-Gordon equation. Physica D 19, 165 (1986)

21. A. Moradi Marjaneh, A. Askari, D. Saadatmand, S.V. Dmitriev, Extreme values of elastic strain and energy in sine-Gordon multikink collisions. Eur. Phys. J. B 91, 22 (2018). arXiv:1710.10159

22. F.C. Simas, A.R. Gomes, K.Z. Nobrega, J.C.R.E. Oliveira, Suppression of two-bounce windows in kink-antikink collisions. JHEP 09, 104 (2016). arXiv: 1605.05344

23. D. Bazeia, E. Belendryasova, V.A. Gani, Scattering of kinks of the sinh-deformed $\varphi^{4}$ model. Eur. Phys. J. C 78, 340 (2018). arXiv: 1710.04993

24. T.S. Mendonca, H.P. de Oliveira, The collision of two-kinks defects. JHEP 09, 120 (2015). arXiv:1502.03870 [hep-th]

25. R. Arthur, P. Dorey, R. Parini, Breaking integrability at the boundary: the sine-Gordon model with Robin boundary conditions. J. Phys. A 49(16), 165205 (2016). arXiv: 1509.08448 [hep-th]

26. P. Dorey, A. Halavanau, J. Mercer, T. Romanczukiewicz, Y. Shnir, Boundary scattering in the $\phi^{4}$ model. JHEP 05, 107 (2017). arXiv:1508.02329 [hep-th]

27. V.A. Gani, A.M. Marjaneh, A. Askari, E. Belendryasova, D. Saadatmand, Scattering of the double sine-Gordon kinks. Eur. Phys. J. C 78(4), 345 (2018). arXiv: 1711.01918 [hep-th]

28. C. Adam, T. Romanczukiewicz, A. Wereszczynski, The $\phi^{4}$ model with the BPS preserving defect. JHEP 03, 131 (2019). arXiv:1812.04007 [hep-th]

29. R. Rajaraman, Solitons and Instantons (North-Holland, Amsterdam, 1982)

30. A. Vilenkin, E.P.S. Shellard, Cosmic Strings, and Other Topological Defects (Cambridge University Press, Cambridge, 1994)

31. N.S. Manton, P. Sutcliffe. Topological Solitons. Cambridge Monographs on Mathematical Physics (Cambridge University Press, Cambridge, 2004)

32. Y.M. Shnir, Topological and Non-topological Solitons in Scalar Field Theories (Cambridge University Press, Cambridge, 2018)

33. D. Bazeia, W. Freire, L. Losano, R.F. Ribeiro, Topological defects and the trial orbit method. Mod. Phys. Lett. A 17, 1945 (2002). arXiv:hep-th/0205305

34. V.I. Afonso, D. Bazeia, M.A.Gonzalez Leon, L. Losano, J. Mateos Guilarte, Orbit-based deformation procedure for two-field models. Phys. Rev. D 76, 025010 (2007). arXiv:0704.2424 [hep-th]

35. J. Sadeghi, A.R. Amani, A. Pourdarvish, The orbit method solution for the deformed three coupled scalar fields. Can. J. Phys. 86, 1-4 (2008). arXiv:0810.0822 [math-ph]

36. G.P. de Brito, A. Souza de Dutra, Orbit based procedure for doublets of scalar fields and the emergence of triple kinks and other defects. Phys. Lett. B 736, 438 (2014). arXiv:1405.5458 [hept-th]

37. E.B. Bogomolny, Stability of classical solutions. Sov. J. Nucl. Phys. 24, 449 (1976)

38. M. Prasad, C.M. Sommerfield, An exact classical solution for the 't Hooft monopole and the Julia-Zee dyon. Phys. Rev. Lett. 35, 760 (1975)

39. D. Bazeia, L. Losano, J.M.C. Malbouisson, Deformed defects. Phys. Rev. D 66, 101701 (2002). arXiv:hep-th/0209027

40. C.A. Almeida, D. Bazeia, L. Losano, J.M.C. Malbouisson, New results for deformed defects. Phys. Rev. D 69, 067702 (2004). arXiv:hep-th/0405238

41. D. Bazeia, A.S. Inácio, L. Losano, Kinks and domain walls in models for real scalar fields. Int. J. Mod. Phys. A 19, 575 (2004)

42. D. Bazeia, Defects Structures in Field Theory (2005). arXiv:hep-th/0507188
43. G. Flores-Hidalgo, One loop renormalization of soliton quantum mass corrections in $(1+1)$-dimensional scalar field theory models. Phys. Lett. B 542, 282 (2002). arXiv:hep-th/0206047

44. A.R. Aguirre, G. Flores-Hidalgo, A supersymmetric exotic field theory in $(1+1)$ dimensions. One loop soliton quantum mass corrections. JHEP 1812, 082 (2018). arXiv: 1609.07341

45. A. Souza de Dutra, P.E.D. Goulart, Nonlinear two-field models from orbit equation deformations. Phys. Rev. D 84, 105001 (2011)

46. D. Bazeia, J. Menezes, M.M. Santos, Complete factorization of equations of motion in Wess-Zumino theory. Phys. Lett. B 521, 418 (2001). arXiv:hep-th/0110111

47. D. Bazeia, J. Menezes, M.M. Santos, Complete factorization of equations of motion in supersymmetric field theories. Nucl. Phys. B 636, 132 (2002). arXiv:hep-th/0103041

48. H. Katsura, Composite-kink solutions of coupled nonlinear wave equations. Phys. Rev. D 89, 085019 (2014). arXiv:1312.4263

49. D. Bazeia, M.M. Santos, Classical stability of solitons in systems of coupled scalar fields. Phys. Lett. 217A, 28 (1996)

50. D. Bazeia, M.J. Santos, R.F. Ribeiro, Solitons in systems of coupled scalar fields. Phys. Lett. A 208, 84 (1995). arXiv:hep-th/0311265v1

51. D. Bazeia, R.F. Ribeiro, M.M. Santos, Solitons in a class of systems of two coupled real scalar fields. Phys. Rev. E 54, 2943 (1996)

52. D. Bazeia, J.R.S. Nascimento, R.F. Ribeiro, D. Toledo, Soliton stability of two real scalar fields. J. Phys. A Math. Gen. 30, 8157 (1997). arXiv:hep-th/9705224

53. S. Flügge, Practical Quantum Mechanics (Springer, Berlin, 1996)

54. F. Cooper, A. Khare, U. Sukhatme, Supersymmetry and quantum mechanics. Phys. Rep. 251, 267 (1995)

55. M.L. Glasser, Determining the energy levels of composite potential wells. Am. J. Phys. 47, 738 (1979)

56. M.L. Glasser, L.M. Nieto, The energy level structure of a variety of one-dimensional confining potentials and the effects of a local singular perturbation. Can. J. Phys. 93, 1588 (2015). arXiv:1505.04362 [quant-ph]

57. F. Garcia-Moliner, J. Rubio, The quantum theory of one-electron states at surfaces and interfaces. Proc. R. Soc. A 324, 257 (1971)

58. H. Kleinert, I. Mustapic, Summing the spectral representations of Pöschl-Teller and Rosen-Morse fixed energy amplitudes. J. Math. Phys. 33, 643 (1992)

59. G. Delfino, G. Mussardo, Non-integrable aspects of the multifrequency sine-Gordon model. Nucl. Phys. B 516, 675 (1998). arXiv:hep-th/9709028

60. L.A. Ferreira, W.J. Zakrzewski, The concept of quasi-integrability: a concrete example. JHEP 05, 130 (2011). arXiv:1011.2176

61. L.A. Ferreira, P. Klimas, W.J. Zakrzewski, Quasi-integrable deformations of the SU(3) Affine Toda theory. JHEP 05, 065 (2016). arXiv: 1602.02003

62. H. Nastase, M. Stephanov, P. van Nieuwenhuizen, A. Rebhan, Nucl. Phys. B 542, 471 (1999). arXiv:hep-th/9802074

63. M. Shifman, A. Vainshtein, M. Voloshin, Phys. Rev. D 59, 45016 (1999). arXiv:hep-th/9810068v2

64. N. Graham, R.L. Jaffe, Nucl. Phys. B 544, 432 (1999). arXiv:hep-th/9808140v3

65. A. Litvintsev, P. van Nieuwenhuizen, Once more on the BPS bound for the SUSY kink. arXiv:hep-th/0010051v2

66. M. Shifman, A. Yung, Supersymmetric solitons. Rev. Mod. Phys. 79, 1139 (2007). arXiv:hep-th/0703267 